\title{
Equivalence classes and conditional hardness in massively parallel computations
}

\author{
Danupon Nanongkai $^{1,2} \cdot$ Michele Scquizzato $^{3}$ (D)
}

Received: 28 October 2020 / Accepted: 17 December 2021 / Published online: 20 January 2022

(c) The Author(s) 2022

\begin{abstract}
The Massively Parallel Computation (MPC) model serves as a common abstraction of many modern large-scale data processing frameworks, and has been receiving increasingly more attention over the past few years, especially in the context of classical graph problems. So far, the only way to argue lower bounds for this model is to condition on conjectures about the hardness of some specific problems, such as graph connectivity on promise graphs that are either one cycle or two cycles, usually called the one cycle versus two cycles problem. This is unlike the traditional arguments based on conjectures about complexity classes (e.g., $P \neq N P$ ), which are often more robust in the sense that refuting them would lead to groundbreaking algorithms for a whole bunch of problems. In this paper we present connections between problems and classes of problems that allow the latter type of arguments. These connections concern the class of problems solvable in a sublogarithmic amount of rounds in the MPC model, denoted by MPC $(o(\log N))$, and the standard space complexity classes $\mathrm{L}$ and $\mathrm{NL}$, and suggest conjectures that are robust in the sense that refuting them would lead to many surprisingly fast new algorithms in the MPC model. We also obtain new conditional lower bounds, and prove new reductions and equivalences between problems in the MPC model. Specifically, our main results are as follows.

- Lower bounds conditioned on the one cycle versus two cycles conjecture can be instead argued under the $\mathrm{L} \nsubseteq \mathrm{MPC}(o(\log N))$ conjecture: these two assumptions are equivalent, and refuting either of them would lead to $o(\log N)$-round MPC algorithms for a large number of challenging problems, including list ranking, minimum cut, and planarity testing. In fact, we show that these problems and many others require asymptotically the same number of rounds as the seemingly much easier problem of distinguishing between a graph being one cycle or two cycles.

- Many lower bounds previously argued under the one cycle versus two cycles conjecture can be argued under an even more robust (thus harder to refute) conjecture, namely $\mathrm{NL} \nsubseteq \mathrm{MPC}(o(\log N))$. Refuting this conjecture would lead to $o(\log N)$ round MPC algorithms for an even larger set of problems, including all-pairs shortest paths, betweenness centrality, and all aforementioned ones. Lower bounds under this conjecture hold for problems such as perfect matching and network flow.
\end{abstract}

Keywords Massively Parallel Computation · Conditional hardness · Fine-grained complexity

\footnotetext{
A preliminary version of this paper [68] appeared in the proceedings of the 23rd International Conference on Principles of Distributed Systems (OPODIS 2019).

Michele Scquizzato scquizza@math.unipd.it

Danupon Nanongkai danupon@gmail.com

1 University of Copenhagen, Copenhagen, Denmark

2 KTH Royal Institute of Technology, Stockholm, Sweden

3 University of Padova, Padua, Italy
}

\section{Introduction}

The Massively Parallel Computation (MPC) model is arguably the most popular model of computation that captures the essence of several very successful general-purpose frameworks for massively parallel coarse-grained computations on large data sets, such as MapReduce [35], Hadoop [86], Spark [88], and Dryad [54]. The main feature of this model is that a single commodity machine of a large cluster 
cannot store the entirety of the input, but just a sublinear fraction of it. This is an important restriction since we think of the data set as being very large. The computation proceeds in synchronous rounds, and in each of them the machines can exchange data with each other with the sole restriction that no one can send or receive more data than it is capable of storing. The goal is to keep the total number of rounds as low as possible.

This basic model has been intensively investigated in the past decade, mostly from an algorithmic point of viewsee $[7,8,11,12,15,17,19,21,24,27,32,33,42-45,47,48,52,59$, $60,63,65,75,78]$ and references therein. It turns out that many problems can be solved with an MPC algorithm that terminates in $O(\log N)$ rounds, where $N$ denotes the input size, usually by simulating known PRAM algorithms [47,59]. However, designing faster algorithms resisted the efforts of many researchers. Recently, a few works managed to break the $O(\log N)$ barrier by relaxing a bit the constraint on the memory size: specifically, they showed that some graph problems allow for $o(\log N)$-round solutions in the so-called near-linear memory regime, whereby machines have memories of size $\tilde{O}(n)$, where $n$ is the number of nodes in the graph $[11,12,21,33,42] .{ }^{1}$ However, without this kind of relaxations only a few problems are known to admit a $o(\log N)$-round algorithm $[17,27,45,48] .^{2}$ A fundamental question is thus whether many known $O(\log N)$-round algorithms can be complemented with a tight lower bound.

Unfortunately, proving unconditional lower bounds-that is, without any assumptions-seems extremely difficult in this model, as it would imply a breakthrough in circuit complexity: Roughgarden et al. [78] showed that, when enough machines are available, proving any super-constant lower bound for any problem in $\mathrm{P}$ would imply new circuit lower bounds, and specifically would separate $\mathrm{NC}^{1}$ from $\mathrm{P}$-a longstanding open question in complexity theory that is a whisker away from the $P$ versus NP question. This means that the lack of super-constant lower bounds in the MPC model can be blamed on our inability to prove some computational hardness results.

In light of this barrier, the focus shifted to proving conditional lower bounds, that is, lower bounds conditioned on plausible hardness assumptions. One widelybelieved assumption concerns graph connectivity, which, when machines have a memory of size $O\left(n^{1-\epsilon}\right)$ for a constant $\epsilon>0$, is conjectured to require $\Omega(\log n)$ MPC rounds

\footnotetext{
${ }^{1}$ Notice that this relaxes the sublinear constraint on the memory size in the case of sparse graphs.

2 Some algorithms have been analyzed in terms of other parameters, such as the diameter $[8,9,19]$ or the spectral gap [14] of the graph. The round complexity of these algorithms is $o(\log N)$ in some cases, but it remains $\Omega(\log N)$ in general. In this paper we do not consider this kind of parameterized analysis.
}

$[15,59,75,78,87] .{ }^{3}$ The same conjecture is often made even for the special case of the problem where the graph consists of either one cycle or two cycles, usually called one cycle versus two cycles problem. The one cycle versus two cycles conjecture has been proven useful to show conditional lower bounds for several problems, such as maximal independent set, maximal matching [43], minimum spanning trees in low-dimensional spaces [7], single-linkage clustering [87], 2-vertex connectivity [9], generation of random walks [64], as well as parameterized conditional lower bounds [19]. ${ }^{4}$

However, it is not clear whether the one cycle versus two cycles conjecture is true or not, and if not, what its refutation implies. This situation is in contrast with traditional complexity theory, where a refutation of a conjectured relationship between complexity classes would typically imply groundbreaking algorithmic results for a large number of problems; for example, if the $\mathrm{P} \neq \mathrm{NP}$ conjecture fails, then there would be efficient (polynomial-time) algorithms for all problems in $N P$, including a number of "hard" problems. To put it another way, a conjecture like $P \neq N P$ is more robust in the sense that it is extremely hard to refute-doing so requires a major algorithmic breakthrough. The goal of this paper is to explore conjectures of this nature in the MPC model.

\subsection{Summary of contributions}

In this paper we show many connections between problems and classes of problems that lead to more robust conjectures for the MPC model. In particular, we study the connections between the class of problems solvable in a sublogarithmic amount of rounds in the MPC model with $O\left(N^{1-\epsilon}\right)$ memory per machine for some constant $\epsilon \in(0,1)$ and up to polynomially many machines, denoted by $\operatorname{MPC}(o(\log N))$, and the standard space complexity classes $L$ and NL. (Recall that $L$ and $N L$ are the classes of decision problems decidable in logarithmic space on deterministic and nondeterministic Turing machines, respectively.) The connection between MPC and these complexity classes is enabled by a recent result showing how Boolean circuits can be efficiently simulated in the MPC model. In short, we present a set of observations and reductions that suggest that $\mathrm{L} \nsubseteq \subseteq \mathrm{MPC}(o(\log N))$ and $\mathrm{NL} \nsubseteq \mathrm{MPC}(o(\log N))$ are two robust conjectures that might play crucial roles in arguing lower bounds in the MPC model, as they already imply tight conditional lower bounds for a large number of problems. In particular, with some assump-

\footnotetext{
3 Observe that in the near-linear memory regime this conjecture breaks: graph connectivity can be solved in $O(1)$ MPC rounds [18].

${ }^{4}$ The one cycle versus two cycles problem is usually stated such that, in the case of two cycles, these have $n / 2$ nodes each. However, we observe that all the mentioned conditional lower bounds hold also when the two cycles may have arbitrary lengths.
} 
tions on the total amount of memory (equivalently, machines) available in the system, we can conclude the following.

1. Robustness: The one cycle versus two cycles conjecture is robust, since it is equivalent to conjecturing that $\mathrm{L} \nsubseteq \mathrm{MPC}(o(\log N))$, and refuting this conjecture requires showing $o(\log N)$-round algorithms for all problems in L. This class includes many important problems such as graph connectivity, cycle detection, and planarity testing.

2. Equivalences: All L-complete problems are equivalent in the sense that they require asymptotically the same number of rounds. This means that the one cycle versus two cycles problem, which is L-complete (see "Appendix A.1"), is equivalent to many seemingly harder problems, such as graph bipartiteness, minimum cut, and formula evaluation (see problems in the bottom ellipse in Fig. 1 for more). This also means that the conjectures on the hardness of graph connectivity and on the hardness of the one cycle versus two cycles problem are equivalent. Additionally, all NL-complete problems and a few others are also equivalent. These problems include $s t$ reachability, all-pairs shortest paths (both the directed and undirected cases) on unweighted graphs, diameter, and betweenness centrality (see problems in the top ellipse in Fig. 1 for more).

3. New conditional lower bounds: Assuming the one cycle versus two cycles conjecture (equivalently, L $\nsubseteq$ $\operatorname{MPC}(o(\log N)))$, there are no $o(\log N)$-round algorithms for all L-hard problems and a few other problems. This implies new conditional lower bounds for more than a dozen of problems, such as betweenness centrality, planarity testing, graph bipartiteness, list ranking, formula evaluation, and densest subgraph (see problems in the big rectangle in Fig. 1 for more). Previously only a few lower bounds were known, e.g., for single-linkage clustering [87] and maximum matching [70]. (Of course, lower bounds for connectivity-related problems are trivially implied by the one cycle versus two cycles conjecture.) Most of our lower bounds are tight (e.g., lower bounds for problems in the ellipses in Fig. 1).

4. A more robust conjecture: For NL-hard problems, we can argue lower bounds under the more robust NL $\nsubseteq$ MPC $(o(\log N))$ conjecture. These problems include perfect matching, single-source shortest paths, diameter, and network flow (see problems in the small rectangle in Fig. 1 for more). Note that, since $\mathrm{L} \subseteq \mathrm{NL}$, the $\mathrm{NL} \nsubseteq$ MPC $(o(\log N))$ conjecture is more robust (i.e., safer, more likely to be true) than its counterpart with $\mathrm{L}$.

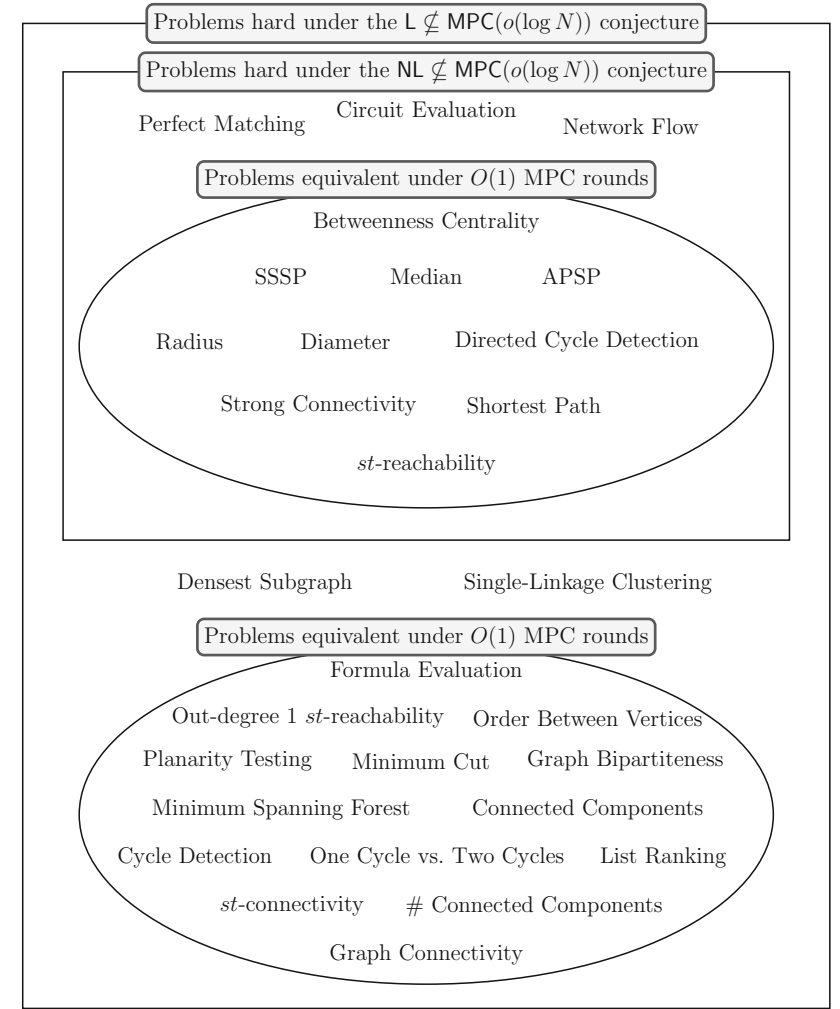

Fig. 1 A classification of the complexity of some prominent problems in the MPC model. Problems in the top ellipse are on unweighted graphs

\subsection{Related work}

Fish et al. [40] were perhaps the first to establish a connection between the MPC model and classical complexity classes. Besides the introduction of a uniform version of the model, they showed that constant-round MPC computations can simulate sublogarithmic space-bounded Turing machines, and then proved strict hierarchy theorems for the MPC model under certain complexity-theoretic assumptions.

Roughgarden et al. [78] discuss connections between the MPC model and Boolean circuits. They show that standard degree arguments for circuits can be applied to MPC computations as well, and specifically that any Boolean function whose polynomial representation has degree $d$ requires $\Omega\left(\log _{s} d\right)$ rounds of MPC using machines with memory $s$. This implies an $\Omega\left(\log _{s} n\right)$ lower bound on the number of rounds for graph connectivity. Perhaps more interestingly, the authors show a barrier for unconditional lower bounds by observing that, if enough machines are available, then proving any super-constant lower bound in the MPC model for any problem in $\mathrm{P}$ would imply new circuit lower bounds, and specifically would separate $\mathrm{NC}^{1}$ from $\mathrm{P}$, thus answering a notorious open question in circuit complexity. This result follows by showing that, with a number of available machines polynomial in the number of input nodes of the 
circuit, $N C^{1}$ circuits can be efficiently simulated in the MPC model. We observe that their argument readily generalizes to show that any bounded fan-in Boolean circuit of depth $d$ and of polynomial size can be simulated in $O(\lceil d / \log s\rceil)$ MPC rounds. Very recently, Frei and Wada [41] prove the same result improving over the amount of machines required for the simulation-from linear to strongly sublinear in the size of the circuit.

Given the difficulty of proving lower bounds for all algorithms, one can (a) prove lower bounds for restricted classes of algorithms, or (b) prove conditional lower bounds: assume one lower bound, and transfer the conjectured hardness to other problems via reductions (with common examples being the theory of NP-hardness and its more recent analogue for problems in $\mathrm{P}$, usually called fine-grained complexity theory). Both paths give a deep understanding and warn us what not to try when designing algorithms.

Within the first line of inquiry, Pietracaprina et al. [74] prove lower bounds for matrix multiplication algorithms that compute all the $n^{3}$ elementary products (thus ruling out Strassen-like algorithms). Similar kinds of limitations are required by Beame et al. [15], Jacob et al. [55], Im and Moseley [51], and Assadi and Khanna [13] to prove lower bounds for $s t$-connectivity, list ranking, graph connectivity, and maximum coverage, respectively. Of a similar flavor are the results of Afrati et al. [3], who show, for a fixed number of rounds (usually a single round), space-communication tradeoffs.

Within the second line of inquiry fall $[7,9,64,87]$, which use the conjecture on the hardness of graph connectivity as a hardness assumption for proving conditional lower bounds for other problems such as minimum spanning trees in low-dimensional spaces, single-linkage clustering, 2-vertex connectivity, and generating random walks, respectively. Recently, Ghaffari et al. [43] present conditional lower bounds for other key graph problems such as constantapproximate maximum matching, constant-approximate vertex cover, maximal independent set, and maximal matching. Their lower bounds also rest on the hardness of graph connectivity, hold for a wide class of MPC algorithms, and are obtained by applying a new general framework (later revised in [31]) that allows one to lift (unconditional) lower bounds from the classical LOCAL model of distributed computing to the MPC model. Assuming the same conjecture, Behnezhad et al. [19] show a parameterized lower bound of $\Omega(\log D)$ for identifying connected components in graphs of diameter $D$. By observing that a couple of specific $N C^{1}$ reductions can be simulated in $O$ (1) MPC rounds, Dhulipala et al. [36] show that if a variant of graph connectivity on batch-dynamic graphs can be solved within a certain amount of rounds, so can all the problems in P. A conditional lower bound following a different kind of argument is given by Andoni et al. [8], who show that an $n^{o(1)}$-round MPC algorithm that answers $O(n+m)$ pairs of reachability queries in directed graphs with $n$ nodes and $m$ edges can be simulated in the RAM model yielding faster Boolean matrix multiplication algorithms. Very recently, Chung et al. [28] show, using techniques from the data structures and cryptography literature, that there exist functions whose computation, assuming the validity of a popular methodology for designing cryptographic constructions, is essentially not parallelizable in the MPC model.

Several other models have been developed in the quest to establish rigorous theoretical foundations of (massively) parallel computing, with the PRAM being one of the most investigated. The MPC model is more powerful than the PRAM since PRAM algorithms can be simulated in the MPC model with constant slowdown [47,59], and some problems (such as sorting or evaluating the XOR function) can be solved much faster in the MPC model.

Valiant's bulk-synchronous parallel (BSP) model [83] anticipated many of the features of MPC-type computations, such as the organization of the computation in a sequence of synchronous rounds (originally called supersteps). Several papers (e.g., $[2,22,46,67,80])$ explored the power of this model by establishing lower bounds on the number of supersteps or on the communication complexity required by BSP computations, where the latter is defined as the sum, over all the supersteps of an algorithm, of the maximum number of messages sent or received by any processor. Lower bounds on the number of supersteps are usually of the form $\Omega\left(\log _{h} N\right)$, where $h$ is the maximum number of messages sent or received by any processor in any superstep.

Another model aiming at serving as an abstraction for modern large-scale data processing frameworks is the $k$ machine model [61]. Inspired by message-passing models in distributed computing, the $k$-machine model features $k$ available machines, and in each round any pair of machines is allowed to communicate using messages of a given size. Hard bounds on the point-to-point communication lead to very strong round lower bounds in this model $[61,71,72]$.

The congested clique (see, e.g., [37]) is a model for network computations bearing some similarities with the MPC model. On one hand, algorithms for this model can be simulated in the MPC model - under some specific conditions on the size of the local memories $[18,42,50]$. On the other hand, analogously to the MPC model, proving a super-constant unconditional lower bound in the congested clique for a problem in NP would imply better circuit size-depth tradeoffs for such a problem than are currently known [37]. This induced further investigations of the model under the lens of complexity theory [62].

Recently, a variant of the MPC model called Adaptive Massively Parallel Computation (AMPC) model has been introduced with the main motivation of alleviating the (conditional) hardness results of the original model. The AMPC 
model extends the MPC model by storing all the messages sent within a round in a distributed hash table; in the following round, each machine can read arbitrary values from this hash table, subject to the same constraints on the amount of communication as in the original model. This extension allows for new graph algorithms with much lower-usually constant-round complexities compared to the best-known solutions in the MPC model [20].

\section{Preliminaries}

\subsection{The MPC model}

The Massively Parallel Computation (MPC) model is a theoretical abstraction capturing the main distinguishing aspects of several popular frameworks for the parallel processing of large-scale datasets. It was introduced by Karloff, Suri, and Vassilvitskii [59], and refined in subsequent work [7,15,47].

In this model the system consists of $p$ identical machines (processors), each with a local memory of size $s$. If $N$ denotes the size of the input, then $s=O\left(N^{1-\epsilon}\right)$ for some constant $\epsilon>0$, and the total amount of memory available in the system is $p \cdot s=O\left(N^{1+\gamma}\right)$ for some constant $\gamma \geq 0$. (Clearly, $p \cdot s \geq N$ must also hold.) The space size is measured by words, each of $\Theta(\log N)$ bits. Initially, the input is adversarially distributed across the machines. The computation proceeds in synchronous rounds. In each round, each machine performs some computation on the data that resides in its local memory, and then, at the end of the round, exchanges messages with other machines. The total size of messages sent or received by each machine in each round is bounded by $s .{ }^{5}$ The goal is to minimize the total number of rounds.

For problems defined on graphs, the input size $N$ is equal to $n+m$, where $n$ is the number of nodes of the graph and $m$ is the number of edges. When considering graph problems, in this paper we assume $s=O\left(n^{1-\epsilon}\right)$. This regime of memory size, usually called strongly sublinear memory regime, is always in compliance with the aforementioned constraint on the size of the local memory, even when graphs are sparse, for which the constraint is the most restrictive.

The value of parameter $\epsilon$ can be chosen by the end user. In particular, when solving problem $A$ on input instance $I$ through a reduction to problem $B$ on input instance $I^{\prime}$ of increased size, a call to the procedure for $B$ should set the value of this parameter to a constant $\epsilon^{\prime} \in(0,1)$ such that $\left|I^{\prime}\right|^{1-\epsilon^{\prime}}=O\left(|I|^{1-\epsilon}\right)$.

Since we want to relate the MPC model to classical complexity classes, one must make sure that the model is uniform,

\footnotetext{
5 This means that there is no computation performed on the fly on incoming data.
}

by which we mean, roughly speaking, that the same algorithm solves the problem for inputs of all (infinitely many) sizes. Fish et al. [40] dealt with this issue observing that Karloff et al.'s original definition of the model [59] is non-uniform, allowing it to decide undecidable languages, and thus by reformulating the definition of the model to make it uniform. Building on that reformulation, and letting $f: \mathbb{N} \rightarrow \mathbb{R}^{+}$be a function, we define the class $\operatorname{MPC}(f(N))$ to be the class of problems solvable in $O(f(N))$ MPC rounds by a uniform family of MPC computations.

\subsection{Circuit complexity background}

In this section we review the Boolean circuit model of computation. An $n$-input, $m$-output Boolean circuit $C$ is a directed acyclic graph with $n$ sources (i.e., nodes with no incoming edges), called input nodes, and $m$ sinks (i.e., nodes with no outgoing edges). All non-source nodes are called gates, and are labeled with one among AND, OR, or NOT. The fan-in of a gate is the number of its incoming edges. The size of $C$ is the total number of nodes in it. The depth of $C$ is the number of nodes in the longest path in $C$.

Note that to decide an entire language, which may contain inputs of arbitrary lengths, we need a family of Boolean circuits, one for each input length. In other words, the Boolean circuit is a natural model for non-uniform computation. When we want to establish relationships between circuit classes and standard machine classes, we need to define uniform circuit classes, with a restriction on how difficult it can be to construct the circuits. The usual notion of uniformity in this case is that of logspace-uniformity: a family of circuits $\left\{C_{n}\right\}_{n \in \mathbb{N}}$ is logspace-uniform if there is an implicitly log-space computable function mapping $1^{n}$ to the description of the circuit $C_{n}$, where implicitly log-space computable means that the mapping can be computed in logarithmic space-see next section for the definition of logarithmic space.

Definition 1 ([10,81]) For $i \geq 1, N C^{i}$ is the class of languages that can be decided by a logspace-uniform family of Boolean circuits with a polynomial number of nodes of fan-in at most two and $O\left(\log ^{i} n\right)$ depth. The class NC is $\cup_{i \geq 1} \mathrm{NC}^{i}$.

The complexity classes $\mathrm{AC}^{i}$ and $\mathrm{AC}=\cup_{i \geq 0} \mathrm{AC}^{i}$ are defined exactly as $N C^{i}$ and $N C$ except that gates are allowed to have unbounded fan-in. Hence, for every $i \in \mathbb{N}, N C^{i} \subseteq A C^{i}$. By replacing gates with large fan-in by binary trees of gates each with fan-in at most two, we also have $\mathrm{AC}^{i} \subseteq \mathrm{NC}^{i+1}$.

\subsection{Space complexity background}

Space complexity measures the amount of space, or memory, necessary to solve a computational problem. It serves as a further way of classifying problems according to their 
computational difficulty, and its study has a long tradition, which brought several deep and surprising results.

Particularly relevant to this paper are some low-space complexity classes, and specifically, classes of problems that can be solved with sublinear memory. In order for this to make sense-sublinear space is not even enough to store the input - one must distinguish between the memory used to hold the input and the working memory, which is the only memory accounted for. Formally, we shall modify the computational model, introducing a Turing machine with two tapes: a read-only input tape, and a read/write working tape. The first can only be read, whereas the second may be read and written in the usual way, and only the cells scanned on the working tape contribute to the space complexity of the computation. Using this two-tape model, one can define the following complexity classes.

Definition 2 ([10,81]) $L$ is the class of languages that are decidable in logarithmic space on a deterministic Turing machine. NL is the class of languages that are decidable in logarithmic space on a nondeterministic Turing machine.

Informally, logarithmic space is sufficient to hold a constant number of pointers into the input and counters of $O(\log N)$ bits ( $N$ is the length of the input), and a logarithmic number of boolean flags.

As for other complexity classes, problems complete for $\mathrm{L}$ or NL are defined to be the ones that are, in a certain sense, the most difficult in such classes. To this end, we first need to decide on the kind of reducibility that would be appropriate. Polynomial-time reducibility would not be very useful because $\mathrm{L} \subseteq \mathrm{NL} \subseteq \mathrm{P}$, which implies that every language in $\mathrm{L}$ (resp., NL), except $\emptyset$ and $\Sigma^{*}$, would be L-complete (resp., $\mathrm{NL}$-complete). Hence we need weaker versions of reduction, ones that involve computations corresponding to sub-classes of $L$ and NL. One notion of reducibility that makes sense for the class $L$ is that of $N C^{1}$ reducibility [30], where $N C^{1}$ is the class of languages decidable in logarithmic depth by a uniform family of Boolean circuits of bounded fan-in.

Definition 3 A language $B$ is L-complete if (1) $B \in \mathrm{L}$, and (2) every $A$ in $\mathrm{L}_{\text {is }} \mathrm{NC} C^{1}$ reducible to $B$.

$N C^{1}$ reducibility has been defined in [29]. In the literature reductions of even-lower level than $N \mathrm{NC}^{1}$ are used to identify meaningful notions of L-completeness. Examples are projections and first-order reductions. For example, the class first-order logic, denoted as FO, equals the complexity class $A C^{0}$, and since $A C^{0} \subset N C^{1}$, a first-order reduction is strictly stronger than an $\mathrm{NC}^{1}$ reduction.

A good choice for the class NL is to use log-space reductions, that is, reductions computable by a deterministic Turing machine using logarithmic space-specifically, in a log-space reduction all the desired bits of the output function can be decided in logarithmic space; see [10,81] for a more formal definition of log-space reducibility.

Definition 4 ([10,81]) A language $B$ is NL-complete if (1) $B \in \mathrm{NL}$, and (2) every $A$ in NL is log-space reducible to $B$.

Following standard terminology we say that a language is L-hard (under NC ${ }^{1}$ reductions) (resp., NL-hard (under log-space reductions)) if it merely satisfies condition (2) of Definition 3 (resp., Definition 4).

In "Appendix A" we recall some known results on the space complexity of several fundamental problems.

\section{Massively parallel computations and space complexity classes}

In this section we recall a recent result showing that Boolean circuits can be efficiently simulated in the MPC model, and then we build on it to derive new results and conjectures.

\subsection{Efficient circuit simulation in the MPC model}

We now recall the main result in [41] which, roughly speaking, says that any bounded fan-in Boolean circuit of depth $d$ and of polynomial size can be simulated in $O(\lceil d / \log s\rceil)$ MPC rounds. This result is already implicit in [78], where it is achieved by a simple simulation whereby each gate of the circuit is associated with a machine whose responsibility is to compute the output of the gate. This requires the availability of a number of machines linear in the size of the circuit. Very recently, Frei and Wada [41] came up with a more sophisticated strategy, which uses only a strongly sublinear amount of machines. Their strategy employs two distinct simulations: for $\mathrm{NC}^{1}$ circuits they exploit Barrington's wellknown characterization of $\mathrm{NC}^{1}$ in terms of bounded-width polynomial-size branching programs, and thus simulate such branching programs in a constant number of rounds; for the higher levels of the NC hierarchy, the Boolean circuits themselves are directly simulated, suitably dividing the computation into the simulation of sub-circuits of depth $O(\log n)$, each to be accomplished in $O(1)$ rounds.

The authors work in the original model of Karloff et al. [59], but their result seamlessly applies in the refined MPC model.

Theorem 1 ([41]) Let DMPC ${ }^{i}$ denote the class of problems solvable by a deterministic MPC algorithm in $O\left(\log ^{i} N\right)$ rounds with $O\left(N^{1-\epsilon}\right)$ local memory per machine and $O\left(N^{2(1-\epsilon)}\right)$ total memory. Then,

$N C^{i+1} \subseteq D M P C^{i}$

for every $i \in \mathcal{N}$ and for every $\epsilon \in(0,1 / 2)$. (When $i=0$, the result holds also for $\epsilon=1 / 2$.) 
Setting $i=0$, we have the following.

Corollary 1 The class $N C^{1}$ can be simulated in $O(1) M P C$ rounds with $O\left(N^{1-\epsilon}\right)$ local memory per machine and $O\left(N^{2(1-\epsilon)}\right)$ total memory, for any constant $\epsilon \in(0,1 / 2]$.

Since $N C^{1} \subseteq \mathrm{L} \subseteq \mathrm{NL} \subseteq N C^{2}$ (see, e.g., [73]), an immediate by-product of Theorem 1 is that some standard space complexity classes can be efficiently simulated in the MPC model.

Corollary 2 The class $N C^{2}$, and thus the classes $L$ and NL, can be simulated in $O(\log N) M P C$ rounds with $O\left(N^{1-\epsilon}\right)$ local memory per machine and $O\left(N^{2(1-\epsilon)}\right)$ total memory, for any constant $\epsilon \in(0,1 / 2)$.

Corollary 2 implies that many important problems, e.g. most of those listed in "Appendix A" (excluding those not known to be in $\mathrm{L}$ or NL, such as perfect matching), can be solved in $O(\log N)$ MPC rounds.

\subsection{New consequences of circuit simulations}

In this section we discuss new consequences of the fact that the MPC model is powerful enough to efficiently simulate general classes of Boolean circuits.

Theorem 2 Consider the MPC model where the size of the local memory per machine is $O\left(N^{1-\epsilon}\right)$ for some constant $\epsilon \in(0,1 / 2]$, and assume that $\Omega\left(N^{2(1-\epsilon)}\right)$ total memory is available. Let $f: \mathbb{N} \rightarrow \mathbb{R}^{+}$be a function. Then, if any L-hard problem can be solved in $O(f(N))$ MPC rounds, so can all the problems in the class L. Moreover, either all L-complete problems can be solved in $O(f(N)) M P C$ rounds, or none of them can.

Proof Both claims follow directly from the definitions of Lhardness and L-completeness, and from Corollary 1 . Let $A$ be an L-hard problem that can be solved in $O(f(N))$ MPC rounds. By definition of $\mathrm{L}$-hardness, every problem in $\mathrm{L}_{\text {is }} \mathrm{NC}^{1}$ reducible to $A$. By assumption, $\epsilon \in(0,1 / 2]$ and $\Omega\left(N^{2(1-\epsilon)}\right)$ total memory is available, and thus, by Corollary 1 , an $\mathrm{NC}^{1}$ reduction can be simulated in $O(1)$ MPC rounds, giving the first claim. Therefore, in particular, if any L-complete problem can be solved in $O(f(N))$ MPC rounds, so can all the other L-complete problems. In other words, either all Lcomplete problems can be solved in $O(f(N))$ MPC rounds, or none of them can.

We remark that in Theorem 2 no assumption is placed on the function $f(N)$, which therefore can be of any form, even a constant. Hence, Theorem 2 says that all the known L-complete problems such as graph connectivity, graph bipartiteness, cycle detection, and formula evaluation, are equivalent in the MPC model, and in a very strong sense: they all require asymptotically the same number of rounds. (Analogous equivalences are common in computer science, e.g., in the theory of NP-completeness and, at a finer-grained level, in the recent fine-grained complexity theory, where equivalence classes of problems within $\mathrm{P}$, such as the APSP class $[84,85]$, are established.) Thus, this simple result provides an explanation for the striking phenomenon that for these well-studied problems we seem unable to break the $O(\log N)$ barrier in the MPC model. It also implies that the conjectures on the hardness of graph connectivity and on the hardness of the one cycle versus two cycles problem are equivalent, at least when $\Omega\left(N^{2(1-\epsilon)}\right)$ total memory is available.

The next theorem provides an even stronger barrier for improvements in the MPC model.

Theorem 3 Consider the MPC model where the size of the local memory per machine is $O\left(N^{1-\epsilon}\right)$ for some constant $\epsilon \in(0,1 / 2]$, and assume that $\Omega\left(N^{2(1-\epsilon)}\right)$ total memory is available. Let $f: \mathbb{N} \rightarrow \mathbb{R}^{+}$be a function. If any L-hard problem can be solved in $O(f(N))$ MPC rounds, then either all NL-complete problems can be solved in $O(f(N)) M P C$ rounds, or none of them can. Moreover, if any NL-hard and any L-hard problem can be solved in $O(f(N))$ MPC rounds, so can all the problems in the class NL.

Proof Let $A$ be an L-hard problem that can be solved in $O(f(N))$ MPC rounds. Then, by Theorem 2, every problem in the class L can be solved in $O(f(N))$ MPC rounds and thus, in particular, every log-space reduction can be computed in $O(f(N))$ MPC rounds. By definition of NLcompleteness, every problem in NL, and thus, in particular, any NL-complete problem, is log-space reducible to any other NL-complete problem, and this proves the first statement.

Let $B$ be an NL-hard problem that can be solved in $O(f(N))$ MPC rounds. By definition of NL-hardness, every problem in NL is log-space reducible to $B$. Since we have just argued that if any L-hard problem can be solved in $O(f(N))$ MPC rounds, so can any log-space reduction, the second statement follows.

Once again, we stress that in Theorem 3 no assumption is placed on the function $f(N)$, which therefore can be of any form, even a constant.

Theorem 3 indicates that, unless $\mathrm{L}=\mathrm{NL}$, in the MPC model the connectivity problem on directed graphs, which is both NL-complete and L-hard, is strictly harder than on undirected graphs in the sense that breaking the current logarithmic barrier, if possible, would be strictly harder.

Notice that we also have the following weaker, but simpler to prove, result: if any problem NL-complete under $N C^{1}$ reductions (such as $s t$-reachability) can be solved in $O(f(N))$ MPC rounds, so can all the problems in the class NL. This follows directly from the definition of NLcompleteness under $\mathrm{NC}^{1}$ reductions and from Corollary 1 . 
Notice also that the result in Theorem 3 can be extended with the same proof to complexity classes wider than $\mathrm{NL}$, such as $\mathrm{NC}^{2}$ or $\mathrm{P}$, for which hardness is defined in terms of $\log$-space reducibility as well.

\subsubsection{New conjectures}

The common belief that problems such as graph connectivity and list ranking cannot be solved in $o(\log N)$ MPC rounds, along with the equivalence result of Theorem 2, justify the following conjecture.

Conjecture 1 No L-hard problem can be solved in $o(\log N)$ MPC rounds with $O\left(N^{1-\epsilon}\right)$ local memory per machine, for any constant $\epsilon \in(0,1)$, not even with a polynomial amount of total memory. Equivalently,

$\mathrm{L} \nsubseteq \mathrm{MPC}(o(\log N))$

We now show the claimed equivalence.

Proposition 1 The two statements in Conjecture 1 are equivalent.

Proof We shall argue that if any of the two statements is wrong, so is the other, and vice versa. Assume $L \subseteq$ $\operatorname{MPC}(o(\log N))$. Then, some L-complete, and hence L-hard, problem is contained in $\operatorname{MPC}(o(\log N))$, that is, it can be solved in $o(\log N)$ MPC rounds. To show the other direction, assume that there exists an L-hard problem that can be solved in $o(\log N)$ MPC rounds with a polynomial amount of total memory. Then, by Theorem 2 , every problem in $\mathrm{L}$ can be solved in $o(\log N)$ MPC rounds, i.e., L $\subseteq \operatorname{MPC}(o(\log N))$. $\square$

We would like to remark that, in light of Theorem 2, Conjecture 1 is totally equivalent to the preceding conjectures on the hardness of graph connectivity or of the one cycle versus two cycles problem $[15,59,75,78,87]$; however, Theorem 2 significantly strengthens the evidence for such conjectures.

Likewise, Theorem 3 provides a justification for the following conjecture.

Conjecture 2 No NL-hard and L-hard problem can be solved in $o(\log N)$ MPC rounds with $O\left(N^{1-\epsilon}\right)$ local memory per machine, for any constant $\epsilon \in(0,1)$, not even with a polynomial amount of total memory. Equivalently,

$\mathrm{NL} \nsubseteq \subseteq \mathrm{MPC}(o(\log N))$

We now show the claimed equivalence.

Proposition 2 The two statements in Conjecture 2 are equivalent.

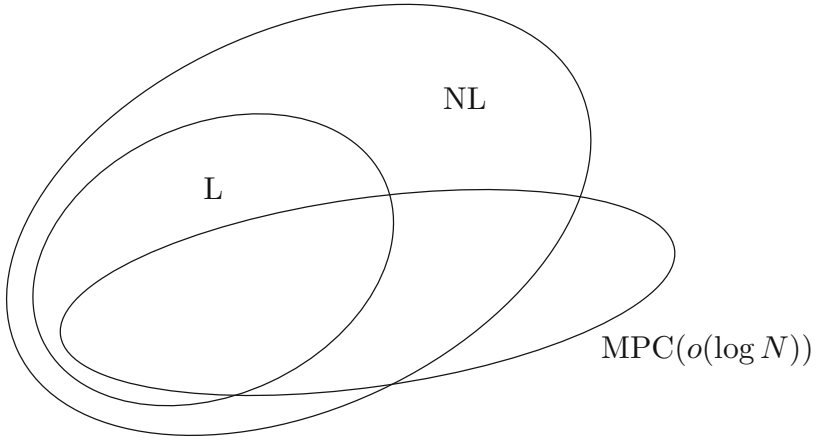

Fig. 2 Conjectured relationships among classes L, NL, and $\operatorname{MPC}(o(\log N))$

Proof We shall argue that if any of the two statements is wrong, so is the other, and vice versa. Assume NL $\subseteq$ $\operatorname{MPC}(o(\log N))$. Then, in particular, $s t$-reachability can be solved in $o(\log N)$ MPC rounds. Since $s t$-reachability is both NL-hard and L-hard, this contradicts the first statement. To show the other direction, assume that there exists an NL-hard and L-hard problem that can be solved in $o(\log N)$ MPC rounds with a polynomial amount of total memory. Then, by Theorem 3, every problem in NL can be solved in $o(\log N)$ MPC rounds, i.e., $\mathrm{NL} \subseteq \mathrm{MPC}(o(\log N))$.

Figure 2 depicts the conjectured relationships among $\mathrm{L}$, $\mathrm{NL}$, and $\mathrm{MPC}(o(\log N))$. Observe that since $\mathrm{L} \subseteq \mathrm{NL}$, Conjecture 1 implies Conjecture 2. Hence, unless $L=N L$, Conjecture 2 is weaker than Conjecture 1, and thus more likely to be true.

We stress that breaking either conjecture would have vast consequences because of the large number of fundamental problems contained in $\mathrm{L}$ and NL. This is somewhat in contrast, e.g., to the Strong Exponential Time Hypothesis (SETH), a popular hardness assumption on the complexity of $k$-SAT used to prove a plethora of conditional lower bounds, especially in the realm of polynomial-time algorithms [84], whose refutation would have more limited algorithmic consequences.

These two conjectures can be used as a base for conditional lower bounds in the MPC model, in the same way as the one cycle versus two cycles conjecture was used as a hardness assumption in $[7,9,19,43,64,87]$.

\section{Reductions and equivalences in massively parallel computations}

In this section we discuss two equivalence classes of problems and some conditional lower bounds in the MPC model. The two equivalence classes both contain problems equivalent to each other under $O(1)$-round MPC reductions and for which the best known upper bound is $O(\log N)$ rounds, but 
differ in terms of the low-space computational complexity characterization of the problems they contain.

As a consequence of the results of Sect. 3, most of these reductions and equivalences follow from known hardness and completeness results for low-space complexity classes such as $\mathrm{L}$ and $\mathrm{NL}$.

We will also show novel, simple reductions and equivalences in the MPC model. Some of such reductions crucially require the availability of up to polynomially many machines (equivalently, a total amount of memory up to polynomial in the input size), which are used to host up to a polynomial number of copies of the input data. The quick creation of so many input replicas can be achieved through the use of a simple two-step broadcast procedure, as shown in the following lemma.

Lemma 1 The input data can be replicated up to a polynomial number of times in $O(1) M P C$ rounds.

Proof Assume that initially all the input data is held by the first $\beta$ consecutively numbered machines. (This is without loss of generality since sorting can be done deterministically in $O$ (1) MPC rounds [47].) We use a basic two-step broadcast procedure to replicate the contents of each machine in $O\left(N^{1-\epsilon}\right)$ other machines. Any polynomial-factor replication can thus be achieved by repeating the procedure a constant amount of times.

Let $c$ be a sufficiently large positive constant. For each $i \in[\beta]$, machine $i$ logically partitions its memory contents in $c N^{1-\epsilon}$ parts, one for each word. Then, machine $i$ sends the $j$-th word to the $\left(\beta+(i-1) c N^{1-\epsilon}+j\right)$-th machine. Finally, each of these machines broadcasts the word received from machine $i$ to all the other machines in the range $\beta+(i-$ 1) $c N^{1-\epsilon}+1, \ldots, \beta+i c N^{1-\epsilon}$, thus yielding a factor- $c N^{1-\epsilon}$ replication of the contents of each machine.

\subsection{An equivalence class for undirected graph connectivity}

In this section we discuss the MPC equivalence class for graph connectivity in undirected graphs. This problem, which asks to determine whether a given undirected graph is connected or not, was one of the first problems to be shown L-hard under (uniform) $\mathrm{NC}^{1}$ reductions [30], and then it was placed in $L$ by the remarkable algorithm of Reingold [76]. Exploiting the results of Sect. 3, we know that one can recycle all the reductions that have been developed in classical complexity theory for showing hardness and completeness for class $L$ in the MPC model as well, since these can all be simulated in $O(1)$ MPC rounds with $O\left(N^{2(1-\epsilon)}\right)$ total memory. This immediately implies that the class of L-complete problems forms an equivalence class in the MPC model as well. Specifically, for example, either all the following problems can be solved with a sublogarithmic MPC algorithm, or none of them can: ${ }^{6}$ graph connectivity, connectivity for promise graphs that are a disjoint union of cycles, st-connectivity, $s t$-reachability for directed graphs of out-degree one, cycle detection, order between vertices, formula evaluation, and planarity testing.

Recycling (some) old SL-completeness results Many more problems can be placed in this MPC equivalence class almost effortlessly: this is the case for some problems complete for the class symmetric logarithmic space (SL), a class defined by Lewis and Papadimitriou [66] to capture the complexity of undirected $s t$-connectivity before this was eventually settled by the breakthrough of Reingold. Completeness in SL is defined in terms of log-space reductions, and $s t$-connectivity is one complete problem for it. Since $L \subseteq$ SL, Reingold's algorithm made these two classes collapse, thus widening the class $L$ with many new problems. However, completeness for $S L$ does not translate into completeness for $L$, since the latter is defined in terms of a lower-level kind of reduction. Luckily, some of the log-space reductions devised to show hardness for SL turn out to be actually stronger than log-space. This is the case, e.g., of testing whether a given graph is bipartite (or, equivalently, 2-colorable), as we show next.

Lemma 2 Graph bipartiteness is equivalent to st-connectivity under $O(1)$-round MPC reductions, with $O\left(n^{1-\epsilon}\right)$ local memory per machine for some constant $\epsilon \epsilon$ $(0,1)$, and $O(n(n+m))$ total memory.

Proof Jones et al. [57] showed that testing whether a graph is non-bipartite is equivalent to $s t$-connectivity under log-space reductions. We will now argue that both reductions can be simulated in $O(1) \mathrm{MPC}$ rounds.

We start by showing that $s t$-connectivity reduces to graph bipartiteness in $O(1)$ MPC rounds. The idea is to make use of the fact that a graph is bipartite if and only if it has no cycle of odd length. Given an instance $G=(V, E), s$, and $t$ of $s t$-connectivity, we build a new graph $G^{\prime}=\left(V^{\prime}, E^{\prime}\right)$ where

$$
\begin{aligned}
V^{\prime}= & \left\{u, u^{\prime}: u \in V\right\} \cup\left\{e, e^{\prime}: e \in E\right\} \\
& \cup\{w\}(\text { with } w \notin V \cup E)
\end{aligned}
$$

and

$$
\begin{aligned}
E^{\prime}= & \left\{\{u, e\},\{e, v\},\left\{u^{\prime}, e^{\prime}\right\},\left\{e^{\prime}, v^{\prime}\right\}: e=\{u, v\} \in E\right\} \cup \\
& \left\{\left\{s, s^{\prime}\right\},\{t, w\},\left\{t^{\prime}, w\right\}\right\} .
\end{aligned}
$$

Then observe that $G^{\prime}$ contains an odd-length cycle, and hence is not bipartite, if and only if $s$ is connected to $t$ in

\footnotetext{
${ }^{6}$ See "Appendix A" for precise definitions of the various problems, as well as for references.
} 
$G$. Nodes and edges of $G^{\prime}$ can be easily generated in $O(1)$ rounds, and stored with $O(n(n+m))$ total memory. Since $\left|V^{\prime}\right|=O\left(n^{2}\right)$, when working with $G^{\prime}$ the size of the local memory is set to $n^{2\left(1-\epsilon^{\prime}\right)}$ where $\epsilon^{\prime} \in(0,1)$ is a constant such that $n^{2\left(1-\epsilon^{\prime}\right)}=O\left(n^{1-\epsilon}\right)$. Then, an $O(f(n))$-round algorithm for graph bipartiteness translates into an $O(f(n))$ round algorithm for $s t$-connectivity.

We now show that graph bipartiteness reduces to $s t$ connectivity in $O(1) \mathrm{MPC}$ rounds. Given an instance $G=$ $(V, E)$, the idea is to construct a new graph $G^{\prime}$ by creating two copies of each node, call them copy 0 and copy 1 , and then for any edge $\{u, v\} \in E$, connecting copy 0 of $u$ to copy 1 of $v$ and vice versa. This can be trivially done in $O(1)$ MPC rounds. It can be observed that $G$ is not bipartite if and only if there is some node $w$ such that copy 0 of $w$ is reachable from copy 1 of $w$. To take care of the phrase "there is some node $w$ ", $n$ copies of $G^{\prime}$ are created and new nodes $s$ and $t$ are introduced. Then $s$ (resp., $t$ ) is connected to copy 0 (resp., copy 1 ) of the $i$-th node in the $i$-th copy of $G^{\prime}$. By Lemma 1 , this can be accomplished in $O(1)$ MPC rounds as well. Then $G$ is not bipartite if and only if there is a path between nodes $s$ and $t$ in this graph.

A good source of problems complete for SL is [6].

From decision to search problems Complexity classes such as $\mathrm{L}$ contain problems phrased as decision problems. Nevertheless, it is often easy to transform a decision problem into its search version - perhaps at the price of a large amount of total memory requirements. As an example, consider the problem called order between vertices (ORD), which, given a directed path specified by giving for each node its successor in the path, and two distinguished nodes $a$ and $b$, asks to determine whether $a$ precedes $b$. ORD is the decision version of list ranking, the problem of obtaining a total ordering from a given successor relation [38]. It is easy to argue the following equivalence.

Lemma 3 List ranking is equivalent to order between vertices under $O(1)$-round MPC reductions, with $O\left(n^{1-\epsilon}\right)$ local memory per machine for some constant $\epsilon \in(0,1)$, and $O\left(n^{3}\right)$ total memory.

Proof Order between vertices trivially reduces to list ranking. We now argue that list ranking is reducible under $O(1)$-round MPC reductions to ORD when there are polynomially many available machines. The reduction is as follows: (1) create $\left(\begin{array}{l}n \\ 2\end{array}\right)$ replicas of the $n$ inputs across the machines; by Lemma 1 this takes $O(1)$ MPC rounds; (2) in parallel, solve ORD for each pair of nodes, one pair for each input replica; (3) each of $n$ designated machines outputs the rank of a distinct node $u$ by counting the number of yes/no outputs for ORD for the pair $(u, v)$, for each $v \neq u$ : doing this is tantamount to doing summation, which can be done in $O(1) \mathrm{MPC}$ rounds by [26] and Corollary 1.

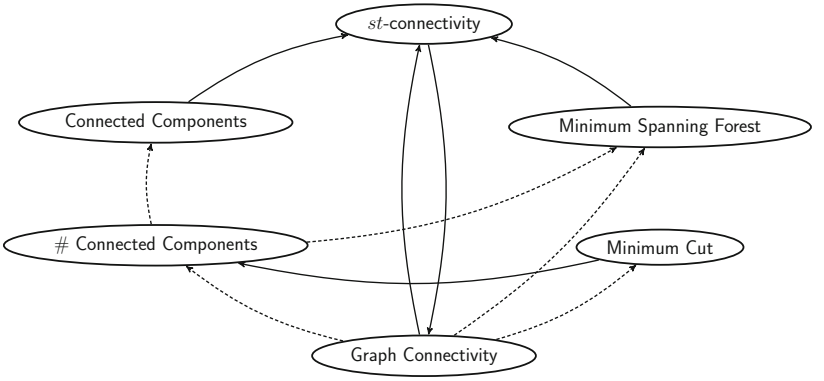

Fig. 3 Constant-round reductions among graph connectivity and related problems. Dashed arrows correspond to trivial reductions

Non-pairwise reductions Sometimes back-and-forth reductions between two problems are not known. In this case their equivalence may nevertheless be established through a series of reductions involving related problems. As an example, we now show that a bunch of problems related to graph connectivity are all equivalent under $O(1)$-round MPC reductions. Besides graph connectivity and $s t$-connectivity, these are determining the connected components of an undirected graph, counting the number of connected components (\# connected components), finding a minimum-weight spanning forest (MSF), and finding a minimum cut. See Fig. 3. Recall that a connected component of an undirected graph is a maximal set of nodes such that each pair of nodes is connected by a path, and it is usually represented by a labeling of nodes such that two nodes have the same label if and only if they are in the same connected component. A minimum spanning forest of a weighted graph is the union of the minimum spanning trees for its connected components. In the minimum cut problem we have to find a partition of the nodes of a graph into two disjoint sets $V_{1}, V_{2}=V \backslash V_{1}$ such that the set of edges that have exactly one endpoint in $V_{1}$ and exactly one endpoint in $V_{2}$ is as small as possible.

Lemma 4 Graph connectivity, st-connectivity, \# connected components, connected components, minimum spanning forest, and minimum cut are all equivalent under $O(1)$-round $M P C$ reductions, with $O\left(n^{1-\epsilon}\right)$ local memory per machine for some constant $\epsilon \in(0,1)$, and $\tilde{O}\left(n^{2} m(n+m)\right)$ total memory.

Proof The reductions from graph connectivity to detecting the number of connected components, to MSF, and to minimum cut are obvious. The reductions from \# connected components to connected components and to MSF are also obvious. We already mentioned that there is a non-obvious low-level equivalence between graph connectivity and $s t$ connectivity, shown by Chandra et al. [26].

Log-space reductions to st-connectivity from MSF and from connected components were given by Nisan and TaShma [69] for showing that the class SL is closed under 
complement. Here we will argue that these reductions can be simulated in $O(1)$ MPC rounds.

We first discuss how to reduce connected components to $s t$-connectivity. The reduction is to evaluate, for each node $s, s t$-connectivity for every other node $t$ in the graph. Then, the label $\ell$ assigned to node $s$ is

$\ell(s)=\min _{t \in V}\{$ ID of node $t$ such that $t$ is connected to $s\}$.

By Lemma 1 and by the fact that the min function can be evaluated in $O(1)$ rounds (by [26] and Corollary 1), this reduction can be accomplished in $O(1)$ MPC rounds.

We now discuss how to reduce MSF to st-connectivity. This is based on the following simple property shown in [69], implicitly used in several other similar reductions [4,8,49,71]: an edge $e=\{u, v\}$ is in the minimum-weight spanning forest if and only if $u$ is not connected to $v$ in the graph made up of all edges having lower weight than $e$. Then, by Lemma 1, in $O(1)$ rounds the input graph can be replicated $m$ times across the available machines, and then testing whether a designated edge $e$ is in the (unique) minimumweight spanning forest of $G$ can be done in parallel for each edge of the graph.

Finally, we discuss how to reduce minimum cut to \# connected components. This is based on the parallelization of Karger's celebrated contraction algorithm [58]—hence this is a randomized reduction, which works with high probability. Recall that Karger's algorithm repeats $O\left(n^{2} \log n\right)$ times the process of contracting randomly chosen edges, one by one, until only two nodes remain. By assumption, we have enough machines to replicate the input graph those many times in $O$ (1) MPC rounds (by Lemma 1) and run the $O\left(n^{2} \log n\right)$ trials in parallel. Identifying the minimum cut from these results can be done in $O(1)$ MPC rounds.

The question is therefore how to run a single time the contraction algorithm. To this end, it is convenient to work with the following equivalent reformulation of the contraction algorithm—see [58, Section 3.1]. First, generate a random permutation of the $m$ edges. Generating a random permutation can be done in $O(1)$ rounds by having each processor take one edge and assign it a score chosen uniformly at random from a sufficiently large range of integers, and then by sorting these scores. Then, imagine contracting edges in the order in which they appear in the permutation until only two nodes remain - this is equivalent to the original formulation of the contraction algorithm. With a sufficiently high probability, a random permutation will yield a contraction to two nodes which determine a particular minimum cut. Then, consider any such permutation. The key property is that it has a prefix such that the set of edges in this prefix induces two connected components (the two sides of the cut), that any prefix which is too short yields more than two connected compo- nents, and that any prefix which is too long yields only one. Hence, with enough machines available, we can determine the correct prefix by examining all the $m$ prefixes of each permutation in parallel.

We can now summarize all the results of this section.

Theorem 4 The following problems are all equivalent under $O(1)$-round MPC reductions, with $O\left(n^{1-\epsilon}\right)$ local memory per machine for some constant $\epsilon \in(0,1)$, and $\tilde{O}\left(n^{2} m(n+m)\right)$ total memory: graph connectivity, connectivity for promise graphs that are a disjoint union of cycles, st-connectivity, st-reachability for directed graphs of out-degree one, cycle detection, order between vertices, formula evaluation, planarity testing, graph bipartiteness, list ranking, \# connected components, connected components, minimum spanning forest, and minimum cut.

Conditional hardness: L-hard problems. Finally, there are problems known to be L-hard, but not known to be in $L$, such as densest subgraph and perfect matching (see "Appendix A"). Since for these problems only one-way reductions from problems in $L$ are known, we don't know whether they are part of the equivalence class of undirected graph connectivity.

\subsection{An equivalence class for directed graph connectivity}

In this section we discuss the MPC equivalence class for graph connectivity in directed graphs. The problem corresponding to $s t$-connectivity in directed graphs is $s t$ reachability, that is, the problem of detecting whether there is a path from a distinguished node $s$ to a distinguished node $t$ in a directed graph. $s t$-reachability is the prototypical complete problem for NL $[10,73,81]$.

By Definition 4, hardness in class NL is defined with respect to log-space reducibility, but we do not know whether $\log$-space computations can be simulated in $o(\log N)$ MPC rounds-in fact, in Section 3 we conjecture they cannot. However, it turns out that many of the known log-space reductions that establish NL-hardness of problems can be simulated in $O$ (1) MPC rounds. This is the case, for example, of the reductions between $s t$-reachability and shortest path, the other canonical example of NL-complete problem which, given an undirected (unweighted) graph, two distinguished nodes $s$ and $t$, and an integer $k$, asks to determine if the length of a shortest path from $s$ to $t$ is $k$.

Lemma 5 Shortest path on unweighted graphs is equivalent to st-reachability under $O(1)$-round MPC reductions, with $O\left(n^{1-\epsilon}\right)$ local memory per machine for some constant $\epsilon \epsilon$ $(0,1)$, and $O(n(n+m))$ total memory. 
Proof We first show that $s t$-reachability can be reduced to shortest path in $O$ (1) MPC rounds. For an integer $k$ we denote the set of integers $\{1,2, \ldots, k\}$ by $[k]$. Given a directed graph $G=(V, E)$ and two designated nodes $s$ and $t$, we create a new (undirected) layered graph $G^{\prime}=\left(V^{\prime}, E^{\prime}\right)$ where

$V^{\prime}=\left\{v_{i}: v \in V, i \in[n]\right\}$

and

$$
\begin{aligned}
E^{\prime}= & \left\{\left\{v_{i}, v_{i+1}\right\}: v \in V, i \in[n-1]\right\} \cup \\
& \left\{\left\{u_{i}, v_{i+1}\right\}:(u, v) \in E, i \in[n-1]\right\} .
\end{aligned}
$$

It is easy to see that there is a directed path from $s$ to $t$ in $G$ if and only if there is a path of length $n-1$ from $s_{1}$ to $t_{n}$ in $G^{\prime}$.

We now show the other direction. Given an undirected graph $G=(V, E)$, two designated nodes $s$ and $t$, and an integer $b \in[n-1]$, we create a new directed layered graph $G^{\prime}=\left(V^{\prime}, E^{\prime}\right)$ where

$V^{\prime}=\left\{v_{i}: v \in V, i \in[b]\right\}$

and

$$
\begin{aligned}
E^{\prime}= & \left\{\left(v_{i}, v_{i+1}\right): v \in V, i \in[b-1]\right\} \cup \\
& \left\{\left(u_{i}, v_{i+1}\right):\{u, v\} \in E, i \in[b-1]\right\} .
\end{aligned}
$$

Then again it is easy to see that the length of a shortest path from $s$ to $t$ is at most $b$ if and only if there is a directed path from $s_{1}$ to $t_{b}$ in $G^{\prime}$. If the length is at most $b$ then one can determine if it is exactly $b$ by repeating the same construction with $b-1$ in place of $b$.

In both directions, nodes and edges of $G^{\prime}$ can be easily generated in $O(1)$ rounds, and stored with $O(n(n+m))$ total memory. Since $\left|V^{\prime}\right| \leq n^{2}$, when working with $G^{\prime}$ the size of the local memory is set to $n^{2\left(1-\epsilon^{\prime}\right)}$ where $\epsilon^{\prime} \in(0,1)$ is a constant such that $n^{2\left(1-\epsilon^{\prime}\right)}=O\left(n^{1-\epsilon}\right)$. Then, an $O(f(n))$ round algorithm for one problem translates into an $O(f(n))$ round algorithm for the other, and vice versa.

There are other NL-complete problems that can be shown to be equivalent under $O(1)$-round MPC reductions. Some examples are directed cycle detection, by a simple adaptation of the preceding reductions, and strong connectivity, which follows from a result in [26]. We suspect that many other logspace reductions are actually (or can easily be translated into) $O$ (1)-round MPC reductions, thus enabling us to enlarge the equivalence class for graph connectivity in directed graphs almost effortlessly by leveraging known results in complexity theory.
When this is not possible, one might have to devise novel reductions. We now do so for some important shortestpath-related problems as well as for some graph centrality problems.

\subsubsection{New fine-grained MPC reductions: constant-round equivalences between graph centrality problems, APSP, and diameter}

In this section we prove a collection of constant-round equivalences between shortest path and many other problems on weighted graphs.

First, some preliminaries. In a graph problem, the input is an $n$-node $m$-edge (directed or undirected) graph $G=$ $(V, E)$ with integer edge weights $w: E \rightarrow\{-M, \ldots, M\}$ where $M=O\left(n^{c}\right)$ for some positive constant $c$. $G$ is assumed to contain no negative-weight cycles. Let $d(u, v)$ denote the (shortest-path) distance from node $u \in V$ to node $v \in V$, that is, the minimum over all paths from $u$ to $v$ of the total weight sum of the edges of the path. If there is no path connecting the two nodes, i.e., if they belong to different connected components, then conventionally the distance is defined to be infinite.

The fundamental all-pairs shortest paths (APSP) problem is to compute $d(u, v)$ for every pair of nodes $u, v \in V$. In the (sequential) RAM model, APSP has long been known to admit an $O\left(n^{3}\right)$ time algorithm. Despite the long history, no algorithm that runs in time $O\left(n^{3-\epsilon}\right)$ for some constant $\epsilon>0$ is known, and it is conjectured that no such algorithm exists $[84,85]$. This conjecture is commonly used as a hardness hypothesis in fine-grained complexity theory to rule out faster algorithms than those currently known for several problems [84]. Beyond such APSP-hardness results, some important problems have been shown to be equivalent to APSP, in the sense that either all such problems admit $O\left(n^{3-\epsilon}\right)$ time algorithms, or none of them do $[1,84,85]$.

These equivalences and most hardness results under the APSP hypothesis rely on a reduction from APSP to the negative triangle problem, which asks whether a graph has a triangle with negative total weight. Although negative triangle can be easily solved in $O$ (1) MPC rounds thanks to Lemma 1, a key building block in the reduction from APSP [85] is a well-known equivalence [39] between APSP and the distance product problem of computing the product of two matrices over the $(\mathrm{min},+)$ semiring (also known as min-plus matrix multiplication); unfortunately, in the reduction from APSP to distance product there are $\lceil\log n\rceil$ of such matrix products (by using the "repeated squaring" strategy), and this takes $O(\log n)$ MPC rounds-which is likely to be best possible, for a reason that will be clear in the next paragraph. Hence in the MPC model we cannot rely on a reduction to negative triangle to prove equivalences to APSP or related 


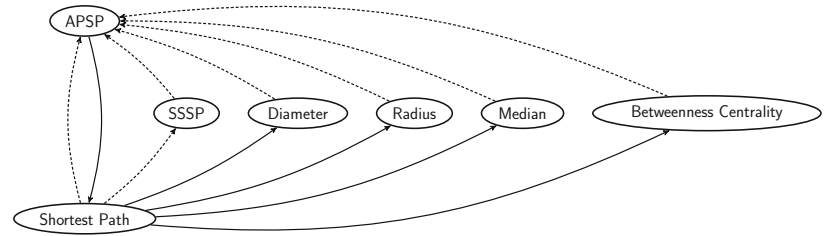

Fig. 4 The constant-round reductions shown in this section. Dashed arrows correspond to trivial reductions

hardness results: we need sublogarithmic fine-grained reductions.

Hence we shall follow a different path, by reducing from the shortest path problem. Given a weighted graph, two distinguished nodes $s$ and $t$, and an integer $k$, shortest path is the problem of determining if the distance of a shortest path from $s$ to $t$ is $k$. This problem is NL-complete, even for undirected and unweighted graphs [23]. (This also explains why the repeated matrix squaring discussed in the previous paragraph is best possible under Conjecture 2.) As we will show shortly, it turns out that shortest path is reducible in $O$ (1) MPC rounds to several fundamental graph problems, including many graph centrality problems defined in terms of shortest paths. Then, by crucially exploiting the availability of many machines, we will argue that APSP is $O(1)$-round reducible to shortest path. Obvious reductions to APSP complete the picture and establish the equivalence of all these problems under $O(1)$-round MPC reductions. See Fig. 4 for a complete summary.

We now formally define the problems we are going to investigate. The eccentricity $\epsilon(v)$ of a node $v$ is the greatest distance between $v$ and any other node. It can be thought of as how far a node is from the node most distant from it in the graph. The diameter of a graph is the greatest distance between any pair of nodes or, equivalently, the maximum eccentricity of any node in the graph, that is,

$\operatorname{diam}(G)=\max _{u \in V} \max _{v \in V} d(u, v)$

The radius of a graph is the minimum eccentricity of any node, that is,

$\operatorname{radius}(G)=\min _{u \in V} \max _{v \in V} d(u, v)$,

and a node with minimum eccentricity is called a center of the graph. The distance sum of a node $u$ is the sum of the distances from $u$ to all the other nodes, that is, $\sum_{v \in V} d(u, v){ }^{7}$ In a (strongly) connected graph, the closeness centrality of a node $u$ is the normalized inverse of its distance sum, that is,

$\mathrm{CC}(u)=\frac{n-1}{\sum_{v \in V} d(u, v)}$.

7 This is sometimes also called the farness of $u$.
A node with maximum closeness centrality, i.e., a node that minimizes the sum of the distances to all other nodes is called a median of the graph, and the value

$\min _{u \in V} \sum_{v \in V} d(u, v)$

is defined as the median of the graph. The betweenness centrality of a node $u$ is defined as

$\mathrm{BC}(u)=\sum_{s, t \in V \backslash\{u\}, s \neq t} \frac{\sigma_{s, t}(u)}{\sigma_{s, t}}$,

where $\sigma_{s, t}$ is the total number of distinct shortest paths from $s$ to $t$, and $\sigma_{s, t}(u)$ is the number of such paths that use $u$ as an intermediate node. Informally, betweenness centrality measures the propensity of a node to be involved in shortest paths.

We start by showing the simple fine-grained equivalence between APSP and shortest path.

Lemma 6 APSP is equivalent to shortest path under $O(1)$ round MPC reductions, with $O\left(n^{1-\epsilon}\right)$ local memory per machine for some constant $\epsilon \in(0,1)$, and $O\left(n^{2}(n+m)\right)$ total memory.

Proof The reduction from shortest path to APSP is obvious. The other direction is also immediate when we have enough machines, and specifically $O\left(n^{2}(n+m)\right)$ total memory: by Lemma 1 we can create $2\left(\begin{array}{l}n \\ 2\end{array}\right)$ copies of the input graph in $O(1)$ MPC rounds, and then in parallel, one pair for each copy, compute the shortest path for each (ordered, if the graph is directed) pair of nodes.

In the following results we will use roughly the same reduction. We start with the problem of determining the diameter of a graph.

Lemma 7 Shortest path is $O(1)$-round MPC reducible to diameter, with $O\left(n^{1-\epsilon}\right)$ local memory per machine for some constant $\epsilon \in(0,1)$, and $O(n+m)$ total memory.

Proof We start with the case of undirected graphs. Given an instance of shortest path, the idea is to alter the input graph by sticking two new and sufficiently long paths to nodes $s$ and $t$, so that the path of largest total weight includes both $s$ and $t$.

This is sufficient if the original graph $G$ is connected; otherwise, the diameter is infinite, and from this information we cannot determine the length of a shortest path from $s$ to $t$. Hence, we shall first make $G$ connected in a way that alters the distance between $s$ and $t$ only if they are not connected in $G$. Since the distance between any two nodes can be at most $(n-1) M$, this can be achieved by adding to the graph a new node $v$ and $n$ edges of weight $n M$ between $v$ and any 


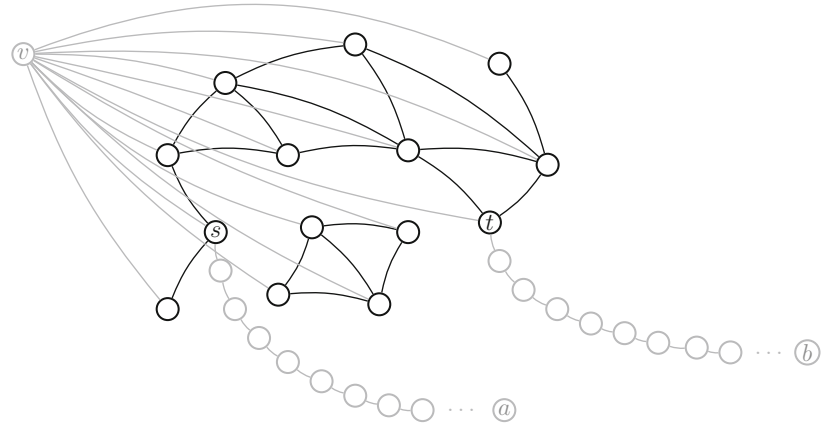

Fig. 5 Reduction from shortest path to diameter. Nodes and edges of the original graph $G$ are in black, whereas nodes and edges added in the reduction are in gray

other node. Then, we append two additional chains to $s$ and $t$, each with $2 n$ edges of weight $M$, and denote this modified graph by $G^{\prime}$. See Fig. 5 .

This reduction can be performed in $O(1)$ MPC rounds, it increases the number of nodes and the number of edges by $O(n)$, and the maximum absolute weight by a factor of $O(n)$. Therefore, any MPC algorithms that runs in $O(f(n, m))$ rounds in the new graph $G^{\prime}$ can be used to solve the original instance $G$ in $O(f(O(n), m+O(n)))=O(f(n, m))$ rounds.

Observe that the diameter of the modified graph $G^{\prime}$ must include the two chains appended to $s$ and $t$. Hence any algorithm for the diameter when executed on graph $G^{\prime}$ always returns $4 n M$ plus the shortest-path distance between $s$ and $t$ in $G^{\prime}$. By construction, the latter quantity, which we denote by $\alpha$, is at most $(n-1) M$ if $s$ and $t$ are connected in $G$, and (exactly) $2 n M$ otherwise. Thus the answer to shortest path is $\alpha$ if the diameter of $G^{\prime}$ is at most $4 n M+(n-1) M$, and infinity otherwise.

In the directed case, we use the same weighted graph $G^{\prime}$ as before, adding one parallel edge for each edge, both with the same weight but with opposite directions. The rest of the algorithm is the same and its analysis is analogous to the undirected case.

Observe that $s t$-connectivity in undirected or directed graphs can also be reduced to diameter, with the same reduction. However, in undirected graphs $s t$-connectivity is only L-hard, while shortest path is NL-hard.

Lemma 8 Shortest path is $O(1)$-round MPC reducible to radius, with $O\left(n^{1-\epsilon}\right)$ local memory per machine for some constant $\epsilon \in(0,1)$, and $O(n+m)$ total memory.

Proof We start with the case of undirected graphs. Given an instance of shortest path, we will construct the graph $G^{\prime}$ of Fig. 5 used in the reduction from shortest path to diameter, and then we will modify $G^{\prime}$ to obtain a new graph $G^{\prime \prime}$ such that $\operatorname{radius}\left(G^{\prime \prime}\right)=\operatorname{diameter}\left(G^{\prime}\right)$.
The graph $G^{\prime \prime}$ is obtained from $G^{\prime}$ by creating a second copy of it, and then by contracting node $b$ of the first copy of $G^{\prime}$ with node $a$ of the second copy of $G^{\prime}$. (Recall that the contraction of a pair of nodes $v_{i}$ and $v_{j}$ of a graph produces a graph in which the two nodes $v_{1}$ and $v_{2}$ are replaced with a single node $v$ such that $v$ is adjacent to the union of the nodes to which $v_{1}$ and $v_{2}$ were originally adjacent.) This reduction can be performed in $O(1)$ MPC rounds, it increases the number of nodes by $O(n)$ and the number of edges by $O(m)$, and the maximum absolute weight by a factor of $O(n)$.

Let $c$ be the node resulting from this contraction. It is easy to see that $c$ is the center of this newly constructed graph $G^{\prime \prime}$ : in fact, by the symmetry of $G^{\prime \prime}$ and by the assignment of the edge weights, any other node has higher eccentricity. Thus, $\operatorname{radius}\left(G^{\prime \prime}\right)=\operatorname{diameter}\left(G^{\prime}\right)$, and hence we can proceed as in the proof of Lemma 7.

In the directed case, we use the same weighted graph $G^{\prime \prime}$ as before, adding one parallel edge for each edge, both with the same weight but with opposite directions. The rest of the algorithm is the same and its analysis is analogous to the undirected case.

Lemma 9 Shortest path is $O(1)$-round MPC reducible to median, with $O\left(n^{1-\epsilon}\right)$ local memory per machine for some constant $\epsilon \in(0,1)$, and $O(n+m)$ total memory.

Proof We start with the case of undirected graphs. Given an instance of shortest path, we construct the graph $G^{\prime \prime}$ as in the reduction from shortest path to radius (see proof of Lemma 8), and compute median $\left(G^{\prime \prime}\right)$. Then, we shall edit $G^{\prime \prime}$ by adding two nodes, $a^{\prime}$ and $b^{\prime}$, as well as two edges, $\left\{a, a^{\prime}\right\}$ and $\left\{b, b^{\prime}\right\}$, both of weight $M$. We call the resulting graph $G^{\prime \prime \prime}$. This reduction can be performed in $O(1)$ MPC rounds, it increases the number of nodes by $O(n)$ and the number of edges by $O(m)$, and the maximum absolute weight by a factor of $O(n)$.

Then, we compute median $\left(G^{\prime \prime \prime}\right)$. Since node $c$ is the median of both $G^{\prime \prime}$ and $G^{\prime \prime \prime}$, we immediately have that

$$
\begin{aligned}
\operatorname{median}\left(G^{\prime \prime \prime}\right)-\operatorname{median}\left(G^{\prime \prime}\right) & =d\left(c, a^{\prime}\right)\left(=d\left(c, b^{\prime}\right)\right) \\
& =\operatorname{radius}\left(G^{\prime \prime}\right)+M \\
& =\operatorname{diameter}\left(G^{\prime}\right)+M,
\end{aligned}
$$

and hence we can proceed as in the proof of Lemma 7.

In the directed case, we use the same weighted graphs $G^{\prime \prime}$ and $G^{\prime \prime \prime}$ as before, adding one parallel edge for each edge, both with the same weight but with opposite directions. The rest of the algorithm is the same and its analysis is analogous to the undirected case.

Now we consider the evaluation of the betweenness centrality of nodes. In contrast to the previous reductions, in the following one we shall create $n$ copies of the reduction graph 
leveraging Lemma 1, and then perform some computation in parallel.

Lemma 10 Shortest path is $O(1)$-round $M P C$ reducible to betweenness centrality, with $O\left(n^{1-\epsilon}\right)$ local memory per machine for some constant $\epsilon \in(0,1)$, and $O(n(n+m))$ total memory.

Proof Once again, we start with the case of undirected graphs. In the directed case we use the same weighted graph adding one parallel edge for each edge, both with the same weight but with opposite directions, with an analogous analysis.

Given an instance of shortest path, we construct the graph $G^{\prime}$ of Fig. 5 as in the reduction from shortest path to diameter. Then, we modify the weights of the edges of $G^{\prime}$ in such a way that exactly one shortest path exists from any node to any other node, and that the length of the original shortest path in $G$ can be easily recovered. To this end, since by assumption the weights of the edges are integers, it is sufficient to increase the weight of each edge of the starting graph $G$ by a real value chosen independently and uniformly at random from the interval $\left[1 / n^{5}, 1 / n^{2}\right]$. (This can be achieved by having each edge pick, independently and uniformly at random, an integer from the set of integers $\left[n^{2}, n^{5}\right]$, hence the probability that any two edges have chosen the same number is at most $\left(\begin{array}{c}m \\ 2\end{array}\right) /\left(n^{5}-n^{2}+1\right)<1 / n^{\Omega(1)}$.) This reduction can be performed in $O(1)$ MPC rounds, it increases the number of nodes and the number of edges by $O(n)$, and the maximum absolute weight by a factor of $O(n)$.

Now we create $n-1$ more copies of this graph, which by Lemma 1 can be done in $O(1)$ MPC rounds, and compute the betweenness centrality of each node of $G$, in parallel on each copy of the graph. Since there is a single shortest path from any node to any other node, the betweenness centrality of a node $u$ is the total number of shortest paths in the graph that use $u$ as an intermediate node. Consider the (unique) shortest path from $s$ to $t$, and let $A$ be the set of its nodes. Let $B=V \backslash A$ be the remaining nodes of $G$. Then observe that (i) for any node $u \in A, \mathrm{BC}(u) \geq 2 n \cdot 2 n$, since $u$ is an intermediate node in each shortest path from any of the $2 n$ nodes of the chain appended to $s$ to any of the $2 n$ nodes of the chain appended to $t$; and (ii) for any node $u \in B, \operatorname{BC}(u) \leq\left(\begin{array}{l}n \\ 2\end{array}\right)$. Hence, to compute the shortest path from $s$ to $t$ in $G$ it is sufficient to consider only the nodes whose betweenness centrality is no less than $4 n^{2}$, and return the sum of the floors of the weights of all edges with both endpoints in this set of nodes. This can be easily done in $O(1)$ MPC rounds.

An immediate consequence of these results is the following.

Proposition 3 Shortest path, SSSP, APSP, diameter, radius, median, and betweenness centrality are all equivalent under
$O$ (1)-round MPC reductions, with $O\left(n^{1-\epsilon}\right)$ local memory per machine for some constant $\epsilon \in(0,1)$, and $O\left(n^{2}(n+m)\right)$ total memory.

Proof The two reductions involving SSSP are obvious. The reduction from diameter (or radius) to APSP is also obvious, since determining the maximum (or minimum) in a set of values can be easily done in $O(1) \mathrm{MPC}$ rounds. The theorem then follows from Lemmas 6, 7, 8, 9, and 10.

It is interesting to observe that this equivalence class includes problems, such as SSSP and APSP, that in the (sequential) RAM model have vastly different complexities, and that an analogous reduction from APSP to diameter in the RAM model seems elusive [1].

We can now summarize all the results of this section.

Theorem 5 The following problems are all equivalent under $O$ (1)-round MPC reductions, with $O\left(n^{1-\epsilon}\right)$ local memory per machine for some constant $\epsilon \in(0,1)$, and $O\left(n^{2}(n+m)\right)$ total memory: st-reachability, strong connectivity, directed cycle detection, unweighted shortest path, unweighted SSSP, unweighted APSP, unweighted diameter, unweighted radius, unweighted median, and unweighted betweenness centrality.

Conditional hardness: problems hard for NL under $O(1)-$ round MPC reductions. Finally, there exist problems known to be hard for NL under $\mathrm{AC}^{0}$, and thus $\mathrm{NC}^{1}$ and $O(1)$-round MPC, reductions, but not known to be in NL. Some examples are perfect matching (even in bipartite graphs), network flow, and circuit evaluation [26]. Since for these problems only one-way reductions from problems in NL are known, we don't know whether they are part of the equivalence class of directed graph connectivity.

\section{Open problems}

The present work can be naturally extended in several directions. One obvious direction is to prove more conditional lower bounds based on the conjectures of this paper, and to show more equivalences between problems.

Several results of this paper, from the connections between MPC computations and space complexity of Section 3 to the reductions of Section 4 , crucially require the availability in the system of a total amount of memory super-linear in the size of the input. These results have no implications for the more interesting case of low total memory-that is, linear or near-linear in the input size. ${ }^{8}$ Hence, it would be interesting to establish equivalence classes and show implications

\footnotetext{
${ }_{8}$ For instance, since the circuit simulation results in $[41,78]$ use superlinear total memory, the barrier for unconditional lower bounds they imply falls for the linear total memory case, for which it is therefore still open the possibility of proving unconditional lower bounds.
} 

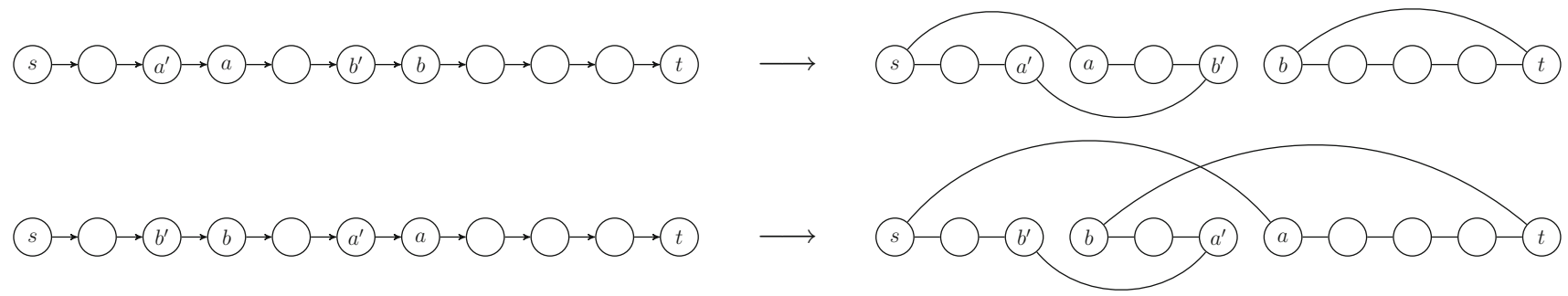

Fig. 6 Reduction from order between vertices to one cycle versus two cycles

that hold under more severe restrictions on the total amount of available memory. (Obtaining low-round reductions with linear or near-linear total space seems to require completely new techniques for several of the problems considered in this paper, though.)

Finally, it is tempting to speculate that improved algorithms for any of the problems discussed in this paper could have significant consequences in other models of computation, such as falsifying some widely-believed conjecture in complexity theory. Identifying new consequences of their falsification would add further weight to the conjectures of this paper.

Acknowledgements This project has received funding from the European Research Council (ERC) under the European Union's Horizon 2020 research and innovation programme under grant agreement No 715672. M. Scquizzato was also partially supported by the University of Padova under grant BIRD197859/19.

Open Access This article is licensed under a Creative Commons Attribution 4.0 International License, which permits use, sharing, adaptation, distribution and reproduction in any medium or format, as long as you give appropriate credit to the original author(s) and the source, provide a link to the Creative Commons licence, and indicate if changes were made. The images or other third party material in this article are included in the article's Creative Commons licence, unless indicated otherwise in a credit line to the material. If material is not included in the article's Creative Commons licence and your intended use is not permitted by statutory regulation or exceeds the permitted use, you will need to obtain permission directly from the copyright holder. To view a copy of this licence, visit http://creativecomm ons.org/licenses/by/4.0/.

\section{Appendix}

\section{A Space complexity of fundamental prob- lems}

Here we report what is known about the space complexity of several fundamental problems. Two good sources of problems complete for $\mathrm{L}$ or $\mathrm{NL}$ are [30,57].

Graph Connectivity: L-complete: L-hard [30, Theorem 3], and in $L$ by virtue of the remarkable algorithm of Reingold
[76]. Remains L-complete for promise graphs that are a disjoint union of cycles [30, Theorem 3].

$s t$-connectivity: L-complete, by virtue of a non-obvious equivalence with graph connectivity under projection reducibility shown by Chandra et al. [26].

$s t$-reachability for directed graphs of out-degree one: The out-degree one version of $s t$-reachability. It is L-complete [56].

Order Between Vertices: Given a directed path, specified by giving for each node its successor in the path, and two distinguished nodes $a$ and $b$,Order Between Vertices (ORD), sometimes also called Path Ordering, asks to determine whether $a$ precedes $b$. ORD is L-complete [38].

Formula Evaluation: A formula is a circuit where each gate has fan-out (out-degree) exactly one, where the underlying algebraic structure is the Boolean algebra. Hence a formula is a circuit whose underlying graph is a tree. Formulas represent computations where the results of subcomputations cannot be used more than once. It is easy to see that Boolean formula evaluation is in L. A seminal paper by Buss shows that Boolean formula evaluation belongs to $\mathrm{NC}^{1}$ [25]. However, for this result it is crucial that the Boolean formula is given as a string (for instance its preorder notation), and not as a tree in pointer representation (e.g., by the list of all edges plus gate types). For the latter representation, the problem is L-complete [16].

Cycle Detection: L-complete, even when the given graph contains at most one cycle [30].

Planarity Testing: Is a given graph planar? Allender and Mahajan [5] showed that this problem is hard for $L$ under projection reducibility (even for graphs of maximum degree 3), and that it lies in SL. Thus, by the result of Reingold [76], planarity testing is L-complete.

Densest Subgraph: Given an undirected graph and a number $k$, is the density of a densest subgraph at least $k$ ? Observe that cycle detection is a special case of this problem with $k=1$, and thus densest subgraph is L-hard.

$s t$-reachability: This is $s t$-connectivity in directed graphs, that is, the problem of detecting whether there is a path from a distinguished node $s$ to a distinguished node $t$ in 
a directed graph. It is denoted STCON, and also known as directed st-connectivity, graph reachability, PATH, or graph accessibility problem (GAP). It is the prototypical complete problem for NL $[10,73,81]$. (This result was first proved by Jones [56], and is implicit in [79], where STCON is called the "threadable maze" problem.) It remains $\mathrm{NL}$-complete for the stronger case of first-order reductions [53]. It is L-hard [66,79], but not known to be in L. In Eulerian directed graphs (i.e., directed graphs where each node has in-degree equal to its outdegree) it is in L [77].

Strong Connectivity: NL-complete: equivalent to $s t$-reachability under $A C^{0}$ reductions [26].

Shortest Path: Given an undirected (unweighted) graph, two distinguished nodes $s$ and $t$, and an integer $k$, the problem of determining if the length of a shortest path from $s$ to $t$ is $k$ is NL-complete [23].

Directed Cycle Detection: Given a directed graph, does it contain a directed cycle? NL-complete [81].

2SAT: NL-complete [73, Theorem 16.3].

NFA/DFA Acceptance: NL-complete [81].

Perfect Matching: NL-hard, even in bipartite graphs, because of a $A C^{0}$ reduction from st-reachability [26]. (Also L-hard, even on $k$-trees [34, Lemma 5.1].) It is a longstanding open question to determine whether perfect matching is in NC (despite some recent substantial progress [82]).

Bipartite Matching, Network Flow: Equivalent under $\mathrm{AC}^{0}$ reductions to bipartite perfect matching [26], and thus NL-hard.

Circuit Evaluation: It is $\mathrm{P}$-complete under $\mathrm{AC}^{0}$ reductions [26], and thus also NL-hard and L-hard.

\section{A.1 L-completeness of the one cycle versus two cycles problem}

In [30, Theorem 3] it is shown that graph connectivity when the given graph is known to be a disjoint union of cycles is Lhard. A careful inspection of the reductions used to establish this result reveals that the problem remains hard even when the graph is known to be made up of either one or three cycles. By reducing from a different problem, we now show that graph connectivity remains hard even when the graph is known to be made up of either one or two cycles. ${ }^{9}$

Proposition 4 Graph connectivity for promise graphs that are either one cycle or two cycles is L-complete.

Proof Membership in $L$ is guaranteed by the algorithm of Reingold [76]. To show L-hardness, we shall exhibit an NC ${ }^{1}$ reduction from order between vertices. Given an instance

\footnotetext{
${ }^{9}$ Graphs are allowed to have parallel edges, that is, cycles with two edges.
}

$(G, a, b)$ for order between vertices, we build a new graph $G^{\prime}$ as follows: (1) the two arcs pointing to $a$ and to $b$, denoted $\left(a^{\prime}, a\right)$ and $\left(b^{\prime}, b\right)$, respectively, are removed, (2) the direction of each of the remaining $n-3$ arcs is discarded, and (3) edges $\{s, a\},\left\{a^{\prime}, b^{\prime}\right\}$, and $\{b, t\}$ are added, where $s$ denotes the source and $t$ the sink of $G$, respectively. See Fig. 6 . This construction is an $\mathrm{NC}^{1}$ reduction. The resulting graph $G^{\prime}$ consists of two cycles if $a$ precedes $b$ in $G$, and of one single cycle otherwise.

\section{References}

1. Abboud, A., Grandoni, F., Vassilevska Williams, V.: Subcubic equivalences between graph centrality problems, APSP and diameter. In: Proceedings of the 26th Annual ACM-SIAM Symposium on Discrete Algorithms (SODA), pp. 1681-1697 (2015)

2. Adler, M. Dittrich, W. Juurlink, B.H.H., Kutylowski, M., Rieping, I.: Communication-optimal parallel minimum spanning tree algorithms. In: Proceedings of the 10th Annual ACM Symposium on Parallel Algorithms and Architectures (SPAA), pp. 27-36 (1998)

3. Afrati, F.N., Sarma, A.D., Salihoglu, S., Ullman, J.D.: Upper and lower bounds on the cost of a Map-Reduce computation. PVLDB 6(4), 277-288 (2013)

4. Ahn, K.J., Guha, S., McGregor, A.: Analyzing graph structure via linear measurements. In: Proceedings of the 23rd Annual ACMSIAM Symposium on Discrete Algorithms (SODA), pp. 459-467 (2012)

5. Allender, E., Mahajan, M.: The complexity of planarity testing. Inf. Comput. 189(1), 117-134 (2004)

6. Àlvarez, C., Greenlaw, R.: A compendium of problems complete for symmetric logarithmic space. Comput. Complex. 9(2), 123-145 (2000)

7. Andoni, A., Nikolov, A., Onak, K., Yaroslavtsev, G.: Parallel algorithms for geometric graph problems. In: Proceedings of the 46th Annual ACM SIGACT Symposium on Theory of Computing (STOC), pp. 574-583 (2014)

8. Andoni, A., Stein, C., Song, Z., Wang, Z., Zhong, P.: Parallel graph connectivity in log diameter rounds. In: Proceedings of the 59th Annual IEEE Symposium on Foundations of Computer Science (FOCS), pp. 674-685 (2018)

9. Andoni, A., Stein, C., Zhong, P.: Log diameter rounds algorithms for 2-vertex and 2-edge connectivity. In: Proceedings of the 46th International Colloquium on Automata, Languages, and Programming (ICALP), pp. 14:1-14:16 (2019)

10. Arora, S., Barak, B.: Computational Complexity: A Modern Approach. Cambridge University Press, Cambridge (2009)

11. Assadi, S., Bateni, M., Bernstein, A., Mirrokni, V.S., Stein, C.: Coresets meet EDCS: algorithms for matching and vertex cover on massive graphs. In: Proceedings of the 30th Annual ACM-SIAM Symposium on Discrete Algorithms (SODA), pp. 1616-1635 (2019)

12. Assadi, S., Chen, Y., Khanna, Y.: Sublinear algorithms for $(\Delta+1)$ vertex coloring. In: Proceedings of the 30th Annual ACM-SIAM Symposium on Discrete Algorithms (SODA), pp. 767-786 (2019)

13. Assadi , S., Khanna, S.: Tight bounds on the round complexity of the distributed maximum coverage problem. In: Proceedings of the 29th Annual ACM-SIAM Symposium on Discrete Algorithms (SODA), pp. 2412-2431 (2018)

14. Assadi, S., Sun, X., Weinstein, O.: Massively parallel algorithms for finding well-connected components in sparse graphs. In: Proceedings of the 38th ACM Symposium on Principles of Distributed Computing (PODC), pp. 461-470 (2019) 
15. Beame, P., Koutris, P., Suciu, D.: Communication steps for parallel query processing. J. ACM 64(6), 1-58 (2017)

16. Beaudry, M., McKenzie, P.: Circuits, matrices, and nonassociative computation. J. Comput. Syst. Sci. 50(3), 441-455 (1995)

17. Behnezhad, S., Brandt, S., Derakhshan, M., Fischer, M., Hajiaghayi, M., Karp, R.M., Uitto, J.: Massively parallel computation of matching and MIS in sparse graphs. In: Proceedings of the 38th ACM Symposium on Principles of Distributed Computing (PODC), pp. 481-490 (2019)

18. Behnezhad, S., Derakhshan, M., Hajiaghayi, M.: Semi-MapReduce meets congested clique. CoRR (2018). arXiv:1802.10297

19. Behnezhad, S., Dhulipala, L., Esfandiari, H., Lacki, J., Mirrokni, V.: Near-optimal massively parallel graph connectivity. In: Proceedings of the 60th IEEE Annual Symposium on Foundations of Computer Science (FOCS), pp. 1615-1636 (2019)

20. Behnezhad, S., Dhulipala, L., Esfandiari, H., Lacki, J., Mirrokni, V.S., Schudy, W.: Parallel graph algorithms in constant adaptive rounds: theory meets practice. Proc. VLDB Endow. 13(13), 3588$3602(2020)$

21. Behnezhad, S., Hajiaghayi, M., Harris, D.G.: Exponentially faster massively parallel maximal matching. In: Proceedings of the 60th IEEE Annual Symposium on Foundations of Computer Science (FOCS), pp. 1637-1649 (2019)

22. Bilardi, G., Scquizzato, M., Silvestri, F.: A lower bound technique for communication in BSP. ACM Trans. Parallel Comput. 4(3), $1-27(2018)$

23. Borodin, A., Cook, S.A., Dymond, P.W., Ruzzo, W.L., Tompa, M.: Two applications of inductive counting for complementation problems. SIAM J. Comput. 18(3), 559-578 (1989)

24. Boroujeni, M., Ehsani, S., Ghodsi, M., Hajiaghayi, M.T., Seddighin, S.: Approximating edit distance in truly subquadratic time: quantum and MapReduce. In: Proceedings of the 29th Annual ACM-SIAM Symposium on Discrete Algorithms (SODA), pp. 1170-1189 (2018)

25. Buss, S.R.: The Boolean formula value problem is in ALOGTIME. In: Proceedings of the 19th Annual ACM Symposium on Theory of Computing (STOC), pp. 123-131 (1987)

26. Chandra, A.K., Stockmeyer, L.J., Vishkin, U.: Constant depth reducibility. SIAM J. Comput. 13(2), 423-439 (1984)

27. Chang, Y.-J., Fischer, M., Ghaffari, M., Uitto, J., Zheng, Y.: The complexity of $(\Delta+1)$ coloring in congested clique, massively parallel computation, and centralized local computation. In: Proceedings of the 38th ACM Symposium on Principles of Distributed Computing (PODC), pp. 471-480 (2019)

28. Chung, K., Ho, K., Sun, X.: On the hardness of massively parallel computation. In: Proceedings of the 32nd ACM Symposium on Parallelism in Algorithms and Architectures (SPAA), pp. 153-162 (2020)

29. Cook, S.A.: A taxonomy of problems with fast parallel algorithms. Inf. Control 64(1-3), 2-22 (1985)

30. Cook, S.A., McKenzie, P.: Problems complete for deterministic logarithmic space. J. Algorithms 8(3), 385-394 (1987)

31. Czumaj, A., Davies, P., Parter, M.: Component stability in lowspace massively parallel computation. In: Proceedings of the 40th ACM Symposium on Principles of Distributed Computing (PODC), pp. 481-491 (2021)

32. Czumaj, A., Davies, P., Parter, M.: Graph sparsification for derandomizing massively parallel computation with low space. ACM Trans. Algorithms 17(2), 1-27 (2021)

33. Czumaj, A., Lacki, J., Madry, A., Mitrovic, S., Onak, K., Sankowski, P.: Round compression for parallel matching algorithms. SIAM J. Comput. 49(5), STOC18-1 (2020)

34. Das, B., Datta, S., Nimbhorkar, P.: Log-space algorithms for paths and matchings in $k$-trees. Theory Comput. Syst. 53(4), 669-689 (2013)
35. Dean, J., Ghemawat, S.: MapReduce: simplified data processing on large clusters. Commun. ACM 51(1), 107-113 (2008)

36. Dhulipala, L., Durfee, D., Kulkarni, J., Peng, R., Sawlani, S., Sun, X.: Parallel batch-dynamic graphs: algorithms and lower bounds. In: Proceedings of the 31st Annual ACM-SIAM Symposium on Discrete Algorithms (SODA), pp. 1300-1319 (2020)

37. Drucker, A., Kuhn, F., Oshman, R.: On the power of the congested clique model. In: Proceedings of the 2014 ACM Symposium on Principles of Distributed Computing (PODC), pp. 367-376 (2014)

38. Etessami, K.: Counting quantifiers, successor relations, and logarithmic space. J. Comput. Syst. Sci. 54(3), 400-411 (1997)

39. Fischer, M.J., Meyer, A.R.: Boolean matrix multiplication and transitive closure. In: Proceedings of the 12th Annual IEEE Symposium on Foundations of Computer Science (FOCS), pp. 129-131 (1971)

40. Fish, B., Kun, J., Lelkes, Á.D., Reyzin, L., Turán, G.: On the computational complexity of MapReduce. In: Proceedings of the 29th International Symposium on Distributed Computing (DISC), pp. $1-15(2015)$

41. Frei, F., Wada, K.: Efficient circuit simulation in MapReduce. In: Proceedings of the 30th International Symposium on Algorithms and Computation (ISAAC), pp. 52:1-52:21 (2019)

42. Ghaffari, M., Gouleakis, T., Konrad, C., Mitrovic, S., Rubinfeld, R.: Improved massively parallel computation algorithms for MIS, matching, and vertex cover. In: Proceedings of the 37th ACM Symposium on Principles of Distributed Computing (PODC), pp. 129-138 (2018)

43. Ghaffari, M., Kuhn, F., Uitto, J.: Conditional hardness results for massively parallel computation from distributed lower bounds. In: Proceedings of the 60th IEEE Annual Symposium on Foundations of Computer Science (FOCS), pp. 1650-1663 (2019)

44. Ghaffari, M., Nowicki, K.: Massively parallel algorithms for minimum cut. In: Proceedings of the 39th ACM Symposium on Principles of Distributed Computing (PODC), pp. 119-128 (2020)

45. Ghaffari, M., Uitto, J.: Sparsifying distributed algorithms with ramifications in massively parallel computation and centralized local computation. In:Proceedings of the 30th Annual ACM-SIAM Symposium on Discrete Algorithms (SODA), pp. 1636-1653 (2019)

46. Goodrich, M.T.: Communication-efficient parallel sorting. SIAM J. Comput. 29(2), 416-432 (1999)

47. Goodrich, M.T., Sitchinava, N., Zhang, Q.: Sorting, searching, and simulation in the MapReduce framework. In: Proceedings of the 22nd International Symposium on Algorithms and Computation (ISAAC), pp. 374-383 (2011)

48. Hajiaghayi, M., Seddighin, S., Sun, X.: Massively parallel approximation algorithms for edit distance and longest common subsequence. In: Proceedings of the 30th Annual ACM-SIAM Symposium on Discrete Algorithms (SODA), pp. 1654-1672 (2019)

49. Hegeman, J.W., Pandurangan, G., Pemmaraju, S.V., Sardeshmukh, V.B., Scquizzato, M.: Toward optimal bounds in the congested clique: graph connectivity and MST. In: Proceedings of the 34th ACM Symposium on Principles of Distributed Computing (PODC), pp. 91-100 (2015)

50. Hegeman, J.W., Pemmaraju, S.V.: Lessons from the congested clique applied to MapReduce. Theor. Comput. Sci. 608, 268-281 (2015)

51. Im, S.,Moseley, B.: A conditional lower bound on graph connectivity in MapReduce. CoRR (2019). arXiv: 1904.08954

52. Im, S., Moseley, B., Sun, X.: Efficient massively parallel methods for dynamic programming. In Proceedings of the 49th Annual ACM SIGACT Symposium on Theory of Computing (STOC), pp. 798-811 (2017)

53. Immerman, N.: Descriptive Complexity. Springer, Berlin (1999)

54. Isard, M., Budiu, M., Yu, Y., Birrell, A.,Fetterly, D.: Dryad: distributed data-parallel programs from sequential building blocks. In: Proceedings of the 2007 EuroSys Conference, pp. 59-72 (2007) 
55. Jacob, R., Lieber, T., Sitchinava, N.: On the complexity of list ranking in the parallel external memory model. In: Proceedings of the 39th International Symposium on Mathematical Foundations of Computer Science (MFCS), pp. 384-395 (2014)

56. Jones, N.D.: Space-bounded reducibility among combinatorial problems. J. Comput. Syst. Sci. 11(1), 68-85 (1975)

57. Jones, N.D., Lien, Y.E., Laaser, W.T.: New problems complete for nondeterministic log space. Math. Syst. Theory 10, 1-17 (1976)

58. Karger, D.R.: Global min-cuts in RNC, and other ramifications of a simple min-cut algorithm. In: Proceedings of the 4th Annual ACM-SIAM Symposium on Discrete Algorithms (SODA), pp. 2130 (1993)

59. Karloff, H.J., Suri, S., Vassilvitskii, S.: A model of computation for MapReduce. In: Proceedings of the 21st annual ACM-SIAM Symposium on Discrete Algorithms (SODA), pp. 938-948 (2010)

60. Kiveris, R., Lattanzi, S., Mirrokni, V.S., Rastogi, V., Vassilvitskii, S.: Connected components in MapReduce and beyond. In: Proceedings of the ACM Symposium on Cloud Computing (SoCC), pp. 18:1-18:13 (2014)

61. Klauck, H., Nanongkai, D., Pandurangan, G.,Robinson, P.: Distributed computation of large-scale graph problems. In: Proceedings of the 26th Annual ACM-SIAM Symposium on Discrete Algorithms (SODA), pp. 391-410 (2015)

62. Korhonen, J.H., Suomela, J.: Towards a complexity theory for the congested clique. In: Proceedings of the 30th ACM Symposium on Parallelism in Algorithms and Architectures (SPAA), pp. 163-172 (2018)

63. Kumar, R., Moseley, B., Vassilvitskii, S., Vattani, A.: Fast greedy algorithms in MapReduce and streaming. ACM Trans. Parallel Comput. 2(3), 1-22 (2015)

64. Lacki, J., Mitrovic, S., Onak, K., Sankowski,P.: Walking randomly, massively, and efficiently. In: Proceedings of the 52nd Annual ACM SIGACT Symposium on Theory of Computing (STOC), pp. 364-377 (2020)

65. Lattanzi, S., Moseley, B., Suri, S., Vassilvitskii, S.: Filtering: a method for solving graph problems in MapReduce. In: Proceedings of the 23rd ACM Symposium on Parallelism in Algorithms and Architectures (SPAA), pp. 85-94 (2011)

66. Lewis, H.R., Papadimitriou, C.H.: Symmetric space-bounded computation. Theor. Comput. Sci. 19, 161-187 (1982)

67. MacKenzie, P.D., Ramachandran, V.: Computational bounds for fundamental problems on general-purpose parallel models. In: Proceedings of the 10th Annual ACM Symposium on Parallel Algorithms and Architectures (SPAA), pp. 152-163 (1998)

68. Nanongkai, D., Scquizzato, M.: Equivalence classes and conditional hardness in massively parallel computations. In: Proceedings of the 23rd International Conference on Principles of Distributed Systems (OPODIS), pp. 33:1-33:16 (2019)

69. Nisan, N., Ta-Shma, A.: Symmetric logspace is closed under complement. In: Proceedings of the 27th Annual ACM Symposium on Theory of Computing (STOC), pp. 140-146 (1995)

70. Onak, K.: Personal communication (2019)

71. Pandurangan, G., Robinson, P., Scquizzato, M.: Fast distributed algorithms for connectivity and MST in large graphs. ACM Trans. Parallel Comput. 5(1), 1-22 (2018)

72. Pandurangan, G., Robinson, P., Scquizzato, M.: On the distributed complexity of large-scale graph computations. ACM Trans. Parallel Comput. 8(2), 1-28 (2021)
73. Papadimitriou, C.H.: Computational Complexity. Addison-Wesley, Boston (1994)

74. Pietracaprina, A., Pucci, G., Riondato, M., Silvestri, F., Upfal, E.: Space-round tradeoffs for MapReduce computations. In: Proceedings of the 26th International Conference on Supercomputing (ICS), pp. 235-244 (2012)

75. Rastogi, V., Machanavajjhala, A., Chitnis, L., Sarma, A.D.: Finding connected components in Map-Reduce in logarithmic rounds. In: Proceedings of the 29th IEEE International Conference on Data Engineering (ICDE), pp. 50-61 (2013)

76. Reingold, O.: Undirected connectivity in log-space. J. ACM 55(4), 17:1-17:24 (2008)

77. Reingold, O., Trevisan, L., Vadhan, S.P.: Pseudorandom walks on regular digraphs and the RL vs. L problem. In: Proceedings of the 38th Annual ACM Symposium on Theory of Computing (STOC), pp. 457-466 (2006)

78. Roughgarden, T., Vassilvitskii, S., Wang, J.R.: Shuffles and circuits (on lower bounds for modern parallel computation). J. ACM 65(6), 1-24 (2018)

79. Savitch, W.J.: Relationships between nondeterministic and deterministic tape complexities. J. Comput. Syst. Sci. 4(2), 177-192 (1970)

80. Scquizzato, M.,Silvestri, F.: Communication lower bounds for distributed-memory computations. In: Proceedings of the 31st International Symposium on Theoretical Aspects of Computer Science (STACS), pp. 627-638 (2014)

81. Sipser, M.: Introduction to the Theory of Computation. PWS Publishing Company, Boston (1997)

82. Svensson, O., Tarnawski, J.: The matching problem in general graphs is in quasi-NC. In: Proceedings of the 58th IEEE Annual Symposium on Foundations of Computer Science (FOCS), pp. 696-707 (2017)

83. Valiant, L.G.: A bridging model for parallel computation. Commun. ACM 33(8), 103-111 (1990)

84. Vassilevska Williams, V.: On some fine-grained questions in algorithms and complexity. In Proceedings of the International Congress of Mathematicians 2018 (ICM 2018), pp. 3431-3472 (2018)

85. Vassilevska Williams, V., Williams, R.R.: Subcubic equivalences between path, matrix, and triangle problems. J. ACM 65(5), 1-38 (2018)

86. White, T.: Hadoop: The Definitive Guide. O'Reilly Media, Inc., Sebastopol (2012)

87. Yaroslavtsev, G., Vadapalli, A.: Massively parallel algorithms and hardness for single-linkage clustering under $\ell_{p}$-distances. In: Proceedings of the 34th International Conference on Machine Learning (ICML), pp. 5596-5605 (2018)

88. Zaharia, M., Xin, R.S., Wendell, P., Das, T., Armbrust, M., Dave, A., Meng, X., Rosen, J., Venkataraman, S., Franklin, M.J., Ghodsi, A., Gonzalez, J., Shenker, S., Stoica, I.: Apache Spark: a unified engine for big data processing. Commun. ACM 59(11), 56-65 (2016)

Publisher's Note Springer Nature remains neutral with regard to jurisdictional claims in published maps and institutional affiliations. 